\title{
Advection-Dominated Accretion and the Black Hole Event Horizon
}

\author{
Ramesh Narayan, Jeffrey E. McClintock \\ Harvard-Smithsonian Center for Astrophysics, Cambridge, MA 02138, U.S.A.
}

\begin{abstract}
As the luminosity of an accreting black hole drops to a few percent of Eddington, the spectrum switches from the familiar soft state to a hard state that is well-described by a distended and tenuous advection-dominated accretion flow (ADAF). An ADAF is a poor radiator, and the ion temperature can approach $10^{12} \mathrm{~K}$ near the center, although the electrons are cooler, with their temperature typically capped at $\sim 10^{9-11} \mathrm{~K}$. The foundational papers predicted that the large thermal energy in an ADAF would drive strong winds and jets, as later observed and also confirmed in computer simulations. Of chief interest, however, is the accreting gas that races inward. It carries the bulk of the accretion energy as stored thermal energy, which vanishes without a trace as the gas passes through the hole's event horizon. One thus expects black holes in the ADAF regime to be unusually faint. Indeed, this is confirmed by a comparison of accreting stellar-mass black holes and neutron stars, which reside in very similar transient X-ray binary systems. The black holes are on average observed to be fainter by a factor of $\sim 100-1000$. The natural explanation is that a neutron star must radiate the advected thermal energy from its surface, whereas a black hole can hide the energy behind its event horizon. The case for an event horizon in Sagittarius $A^{*}$, which is immune to caveats on jet outflows and is furthermore independent of the ADAF model, is especially compelling. These two lines of evidence for event horizons are impervious to counterarguments that invoke strong gravity or exotic stars.
\end{abstract}

Key words:

\section{Introduction}

The foundations of our present understanding of advection-dominated accretion were laid out in a series of papers by Narayan \& Yi (1994, 1995a,b, hereafter NY94, NY95a, NY95b), Abramowicz et al. (1995) and Chen et al. (1995), although some ideas were anticipated much earlier by Ichimaru (1977). The specific abbreviation ADAF, which stands for advection-dominated accretion flow, was introduced by Lasota 1 (1996) and has become standard in the

\footnotetext{
Email addresses: rnarayan@cfa.harvard.edu (Ramesh Narayan), jmcclintock@cfa.harvard.edu (Jeffrey E. McClintock).

1 It gives the authors great pleasure to highlight JeanPierre Lasota's research on ADAFs in this contribution to his Festschrift.
}

field. We review here the application of ADAFs to accreting black holes (BHs), and in $\S 4$ we also consider their application to accreting neutron stars.

\section{Accretion Regimes}

The energy equation per unit volume of an accretion flow can be written compactly in the form

$\rho T \frac{d S}{d t} \equiv \rho T\left(\frac{\partial S}{\partial t}+\vec{v} \cdot \vec{\nabla} S\right)=q_{+}-q_{-}$,

where $\rho$ is the density, $T$ is the temperature, $S$ is the entropy per unit mass, $t$ is time, $\vec{v}$ is the flow velocity, and $q_{+}$and $q_{-}$are the heating and cooling rates per unit volume. This equation states that the rate at which the entropy per unit volume of the gas increases is equal to the heating rate minus the 
cooling rate. Since any entropy stored in the gas is advected with the flow, the left-hand side of equation (1) may be viewed as the effective advective cooling rate $q_{\text {adv }}$. Equation (1) can then be rearranged to give

$q_{+}=q_{-}+q_{\mathrm{adv}}$,

which states that the heat energy released by viscous dissipation is partially lost by radiative cooling and partially by advective cooling.

The standard thin accretion disk model (Shakura \& Sunyaev 1973; Novikov \& Thorne 1973; Frank, King \& Raine 2002) corresponds to the case when the accreting gas is radiatively efficient, so that we have

Thin Disk : $\quad q_{-} \gg q_{\text {adv }}, \quad L \sim 0.1 \dot{M} c^{2}$.

Since the gas cools efficiently, the sound speed is much less than the local Keplerian speed $v_{K}$ and the disk is geometrically thin. Also, the disk radiates about a tenth of the rest mass energy of the accreting gas, the precise fraction depending on the $\mathrm{BH}$ spin (see Shapiro \& Teukolsky 1983).

An ADAF corresponds to the opposite regime. Here the gas is radiatively inefficient and the accretion flow is underluminous. Thus, an ADAF is defined by the condition

ADAF : $\quad q_{\text {adv }} \gg q_{-}, \quad L \ll 0.1 \dot{M} c^{2}$.

Some papers in the literature define an ADAF as a flow that corresponds exactly to a self-similar solution described in NY94 (see §3.1). This is a needless restriction. In our opinion, it is more fruitful to employ the general definition of an ADAF as given in equation (4) 2 .

There are two distinct regimes of advectiondominated accretion. The first, the one that we focus on in this article is when the accreting gas is very tenuous and has a long cooling time (NY94; NY95b; Abramowicz et al. 1995). This regime is sometimes referred to as a RIAF - a "radiatively inefficient accretion flow" - and is defined by the condition

$\mathrm{ADAF} / \mathrm{RIAF}: \quad t_{\mathrm{cool}} \gg t_{\mathrm{acc}}$.

$\overline{2}$ Actually, at mass accretion rates for which the flow is close to the boundary between the thin disk and ADAF solutions, we have (by continuity) $q_{\mathrm{adv}} \gtrsim q_{-}$, and the flow is only marginally radiatively inefficient (see Fig. 4). Nevertheless, even here, the two solutions are very distinct from each other.
Here $t_{\text {cool }}$ is the cooling time of the gas and $t_{\text {acc }}$ is the accretion time.

The second regime of advection-dominated accretion is when the particles in the gas have no trouble cooling, but the scattering optical depth of the accretion flow is so large that the radiation is unable to diffuse out of the system. This radiationtrapped regime was briefly discussed by Begelman (1979) and was developed in detail by Abramowicz et al. (1988) in their "slim disk" model. The defining condition for this regime of ADAFs is

ADAF/Slim Disk : $\quad t_{\text {diff }} \gg t_{\text {acc }}$,

where $t_{\text {diff }}$ is the diffusion time for photons.

The present review is devoted exclusively to the ADAF/RIAF form of accretion (see Narayan, Mahadevan \& Quataert 1998b; Kato, Fukue \& Mineshige 1998; Lasota 1999a,b; Quataert 2001; Narayan 2002, 2005; Igumenshchev 2004; Done, Gierlinsky \& Kubota 2007 for reviews emphasizing various aspects of ADAFs). We will henceforth drop the modifier RIAF and refer to these flows simply as ADAFs.

As explained above, an ADAF is by definition very different from the standard thin accretion disk. Correspondingly, it is characterized by very distinct observational signatures. Both ADAFs and thin disks have well-known counterparts in nature.

\section{Properties of ADAFs}

\subsection{Geometry and Kinematics}

In an $\mathrm{ADAF}$, since most of the energy released by viscous dissipation is retained in the gas, the pressure is large, and so is the sound speed: $c_{s} \sim$ $v_{K}$, where the Keplerian velocity $v_{K}(R)$ is equal to $c / \sqrt{2 r}$ with $r=R / R_{S}$ being the radius in Schwarzschild units, $R_{S}=2 G M / c^{2}$. The large pressure has several immediate consequences. First, the accretion flow becomes geometrically thick, with a vertical height $H$ of order the radius $R$ (an ADAF may be viewed as the viscous rotating analog of spherical Bondi accretion). Second, the flow has considerable pressure-support in the radial direction, so the angular velocity becomes subKeplerian. Third, the radial velocity of the gas is relatively large: $v \sim \alpha v_{K}(H / R)^{2} \sim \alpha c / r^{1 / 2}$, where $\alpha \sim 0.1-0.3$ (see below) is the standard dimensionless viscosity parameter (Shakura \& Sunyaev 1973). Fourth, the large radial velocity leads to a 
short accretion time: $t_{\mathrm{acc}}=R / v \sim t_{\mathrm{ff}} / \alpha$, where $t_{\mathrm{ff}}=\left(2 G M / R^{3}\right)^{1 / 2}$ is the free-fall time. Finally, the large velocity and large scale height cause the gas density to be very low, and so the cooling time is very long and the medium is optically thin.

The above properties are nicely illustrated in the self-similar ADAF solution derived by NY94, in which all quantities in the accretion flow behave as power-laws in radius. (A similar solution was obtained by Spruit et al. 1987 in a different context.) NY94 obtained a general solution for arbitrary viscosity parameter $\alpha$, adiabatic index $\gamma$, and advection parameter $3 f \equiv q_{\text {adv }} / q_{+}$. In the limit $\alpha^{2} \ll 1$ (a good approximation) and $f \rightarrow 1$ (radiatively very inefficient flow), the solution simplifies to

$$
\begin{aligned}
v & =-\alpha\left[\frac{\gamma-1}{\gamma-5 / 9}\right] v_{K}=-0.53 \alpha v_{K}, \\
\Omega & =\left[\frac{2(5 / 3-\gamma)}{3(\gamma-5 / 9)}\right]^{1 / 2} \Omega_{K}=0.34 \Omega_{K}, \\
c_{s} & =\left[\frac{2(\gamma-1)}{3(\gamma-5 / 9)}\right]^{1 / 2} v_{K}=0.59 v_{K} .
\end{aligned}
$$

The numerical coefficients on the right correspond to $\gamma=1.5$, a reasonable choice (see Quataert \& Narayan 1999).

The great virtue of the above self-similar solution is that it is analytic and provides an easy and transparent way of understanding all the key properties of an ADAF. Its biggest deficiency is that it is scalefree, which means that it is inappropriate near the inner or outer boundary of the flow. Therefore, for detailed work, one must use global solutions of the ADAF equations that satisfy appropriate boundary conditions (e.g., Abramowicz et al. 1996; Narayan, Kato \& Honma 1997c; Chen, Abramowicz \& Lasota 1997; Manmoto, Mineshige \& Kusunose 1997; Popham \& Gammie 1998; Manmoto 2000). Calculating global solutions is somewhat involved and one may wish to employ some short-cuts (e.g., Yuan, Ma \& Narayan 2008). On the other hand, even the exact global solutions are somewhat limited since they solve a set of height-integrated equations. For greater realism, one might wish to work directly with numerical simulations (e.g., Goldston, Quataert \& Igumenshchev 2005; Noble et al. 2007).

3 By this definition, $f=0$ corresponds to a fully coolingdominated (no advection) flow and $f=1$ corresponds to a fully advection-dominated (no radiative cooling) flow.

\subsection{Thermal Properties}

The ADAF solution is gas pressure dominated. Since $c_{s} \sim v_{K}$, this means the gas temperature is nearly virial. Under normal conditions, gas at such a high temperature will radiate copiously, especially at small radii where the temperature can approach $10^{12} \mathrm{~K}$. Thus, in order to have an ADAF, the accreting gas generally has to be a two-temperature plasma (at least at small radii), with electron temperature $T_{e}$ much less than the ion temperature $T_{i}$ (NY95b). (The only way to avoid this condition is by having an extremely low accretion rate below about $10^{-6}$ of the Eddington rate.) Typical ADAF models have the two temperatures scaling as

$T_{i} \sim 10^{12} \mathrm{~K} / r, \quad T_{e} \sim \operatorname{Min}\left(T_{i}, 10^{9-11} \mathrm{~K}\right)$.

In order for gas in an ADAF to be twotemperature, there must be weak coupling between electrons and ions. Models generally assume that the coupling occurs via Coulomb collisions, which is inefficient at the densities under consideration. Begelman \& Chiueh (1988) investigated whether plasma instabilities might enhance the coupling and drive the plasma rapidly to a single temperature; this would be problematic for the ADAF solution. However, they were unable to identify a clear mechanism. For normal mass accretion rates, the electrons in a two-temperature ADAF will have a thermal energy distribution (but not necessarily the ions, see Mahadevan \& Quataert 1997).

Early work on two-temperature ADAFs assumed that viscous heating acts primarily on the ions; for instance, NY95b took the ratio of electron heating to total heating, $\delta$, to be zero, while Esin, McClintock \& Narayan (1997) assumed $\delta \sim m_{e} / m_{p} \sim$ $10^{-3}$. However, an ADAF does not require $\delta$ to be this small. Because of a degeneracy in model parameters (Quataert \& Narayan 1999), it is possible to have a viable ADAF model with larger values of $\delta$, provided the mass loss parameter $s$, defined in $\S 3.6$, is adjusted. More recent ADAF models (e.g., Yuan, Quataert \& Narayan 2003) typically assume $\delta \sim 0.3-0.5$.

Various attempts have been made to estimate the value of $\delta$ from first principles by considering the effects of reconnection (Bisnovatyi-Kogan \& Lovelace 1997; Quataert \& Gruzinov 1999) or MHD turbulence (Quataert 1998; Blackman 1999; Medvedev 2000). These studies do not agree on a single value of $\delta$, but generally suggest that $\delta$ is likely to be much 
larger than $10^{-3}$. Recently, Sharma et al. (2007) considered heating by the dissipation of pressure anisotropy and showed that $\delta \sim\left(T_{e} / T_{i}\right)^{1 / 2} / 3$.

It should be noted that, even if electrons and ions receive equal amounts of the dissipated energy (i.e., $\delta=0.5$ ), the plasma can still be two-temperature. This is because a large part of the heating in an ADAF is by compression (since the density increases inward). Once $k T \gtrsim m_{e} c^{2}$, which is the case for $r \lesssim$ a few hundred, the electrons become relativistic and have an adiabatic index $\gamma_{e} \sim 4 / 3$, whereas the ions continue to be non-relativistic with $\gamma_{i} \sim 5 / 3$. Since adiabatic heating by compression causes the temperature to scale as $T \propto \rho^{\gamma-1}$, the electrons heat up only as $T_{e} \sim \rho^{1 / 3}$ whereas the ions heat up as $T_{i} \sim \rho^{2 / 3}$. Thus, even in the limiting case of $\delta \sim 0.5$, ADAFs naturally become two-temperature at small radii. For instance, in the ADAF model of Sagittarius $\mathrm{A}^{*}\left(\mathrm{Sgr} \mathrm{A}^{*}\right)$ proposed by Yuan et al. (2003), the authors obtain $T_{e} \sim 0.1 T_{i}$ close to the $\mathrm{BH}$ even though they assumed $\delta=0.55$.

An important property of the ADAF solution is that it is thermally stable (NY95b; Wu \& Li 1996; Kato et al. 1997; Wu 1997). The demonstration of this property was a crucial advance. Two decades earlier, in a seminal paper, Shapiro, Lightman \& Eardley (1976), and after them Rees et al. (1982; 'ion tori'), introduced the idea of a two-temperature accretion flow and derived a hot two-temperature accretion flow solution, the SLE solution. However, that solution turned out to be thermally unstable (Piran 1978). Until the development of the ADAF solution, and the recognition that it is different from the SLE solution, no stable, hot, optically thin solution was available to model the many accretion systems whose spectra (especially in the hard state, see below) demand such a flow.

Chen et al. (1995) and Yuan (2003) have explored the relationships among the ADAF/RIAF, SLE, ADAF/Slim Disk and Thin Disk solutions.

\subsection{Models and Spectra}

The ADAF is a full accretion solution which incorporates consistent dynamics, thermal balance, radiation physics, etc. Therefore, the radial profiles of all gas properties can be calculated self-consistently once we know the values of certain parameters: $\mathrm{BH}$ mass $M$, accretion rate $\dot{M}$, viscosity parameter $\alpha$, pressure parameter $\beta \equiv P_{\text {gas }} /\left(P_{\text {gas }}+P_{\text {mag }}\right)$, adiabatic index $\gamma$ (usually $\sim 1.5$ ), viscous heating pa- rameter $\delta$, advection parameter $f$.

Actually, apart from the system-specific parameters $M$ and $\dot{M}$ (which may be estimated through observation), most of the other parameters are constrained. Under the nearly collisionless conditions expected in an ADAF, the viscosity parameter is moderately enhanced relative to a collisional gas (Sharma et al. 2006). In the case of dwarf novae in the hot state, Smak (1999) estimates $\alpha \sim$ 0.2 , while numerical simulations of the magnetorotational instability give $\alpha \lesssim 0.1$ (Hawley, Gammie $\&$ Balbus 1996). Thus we expect $\alpha \sim 0.1-0.3$ for an ADAF (typical values used in models are $0.2-0.3)$. Numerical simulations further suggest that magnetic fields are generally subthermal, with $P_{\text {mag }} / P_{\text {gas }} \sim 0.1$ (e.g., Hawley et al. 1996), so we expect $\beta \sim 0.9$. By calculating the energy loss via radiation from the hot accretion flow (synchrotron, bremsstrahlung, Compton scattering), $f$ can be obtained self-consistently (e.g., NY95b; Narayan, Barret \& McClintock 1997a; Esin et al. 1997; Yuan et al. 2003). Thus, we have only one poorly constrained parameter: $\delta$. The recent work of Sharma et al. (2007) provides a serviceable prescription even for this parameter. At large radii, where the plasma is effectively one-temperature, their formula gives $\delta \sim 0.3$, while in the energetically important inner region, where the plasma is two-temperature, they find $\delta \sim 0.01-0.1$, depending on model details. The final two parameters are discussed later: the transition radius $R_{\mathrm{tr}}, \S 3.5$, and the wind parameter $s, \S 3.6$.

Since the earliest days of X-ray astronomy, it has been clear that BH binaries (BHBs) have a number of distinct spectral states (see Zdziarski \& Gierlinski 2004; McClintock \& Remillard 2006; Done et al. 2007). The most notable among these are the luminous high soft state, or thermal state, the slightly less luminous low hard state, and the very underluminous quiescent state.

The thermal state is well described by the thin disk model, and a multi-color disk (MCD) blackbody model (e.g., diskbb, Mitsuda et al. 1984; ezdiskbb, Zimmerman et al. 2005; both available in XSPEC, Arnaud et al. 1996) has been successfully used for years to model the X-ray spectra of sources in this state. Recently, fully relativistic versions of the MCD model for arbitrary BH spin (kerrbb, Li et al. 2005; bhspec, Davis \& Hubeny 2006) have been developed, based on the relativistic thin disk model of Novikov \& Thorne (1973). These models provide excellent fits to the X-ray spectra of BHBs in the 


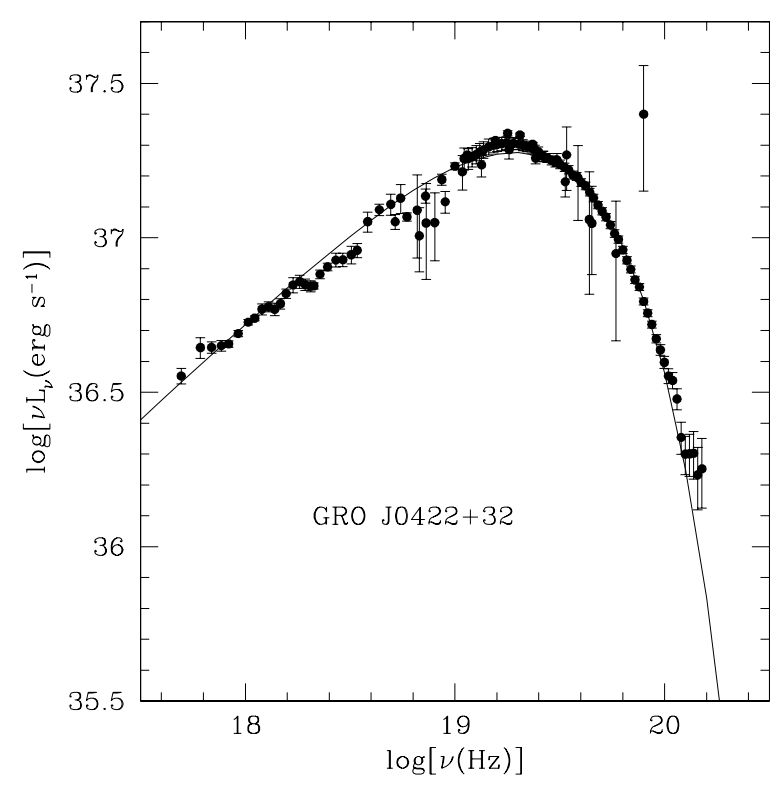

Fig. 1. Combined TTM $(2-20 \mathrm{keV})$, HEXE $(20-200 \mathrm{keV})$, and OSSE (50-600 keV) spectrum of GRO J0422+32 observed between 1992 August 29 and September 2. The solid line is an ADAF model which reproduces the overall luminosity of the source, the power-law slope below the peak and the shape of the high-energy cutoff. The model has a mass accretion rate $\sim 0.1 \dot{M}_{\mathrm{Edd}}$. (From Esin et al. 1998)

thermal state (e.g., McClintock et al. 2006; Davis, Done \& Blaes 2006).

Whereas a satisfactory theoretical model, viz., the thin disk model, was established early on for the thermal state, the hard state was for many years a mystery. Figure 1 shows the spectrum of a typical BHB, GRO J0422+32, in the hard state (Esin et al. 1998). The observations indicate that the accreting gas is very hot, $k T \gtrsim 100 \mathrm{keV}$. The gas must also be optically thin, since optically thick blackbody emission at a temperature of $100 \mathrm{keV}$ would correspond to a luminosity $L=\sigma T^{4} A \gtrsim 10^{46} \mathrm{erg} \mathrm{s}^{-1}$ for any reasonable estimate of the radiating area $A$.

The most natural explanation of the emission in J0422 and other BHBs in the hard state is that it is produced by thermal Comptonization. Until the ADAF model was established, no accretion model could reproduce such a spectrum. (The SLE solution could, but it was unstable.) Indeed, astronomers were reduced to using empirical Comptonization models (Sunyaev \& Titarchuk 1980) in which they postulated a Comptonizing cloud with some arbitrary geometry and parameterized the cloud with an adjustable temperature and an optical depth (e.g.,

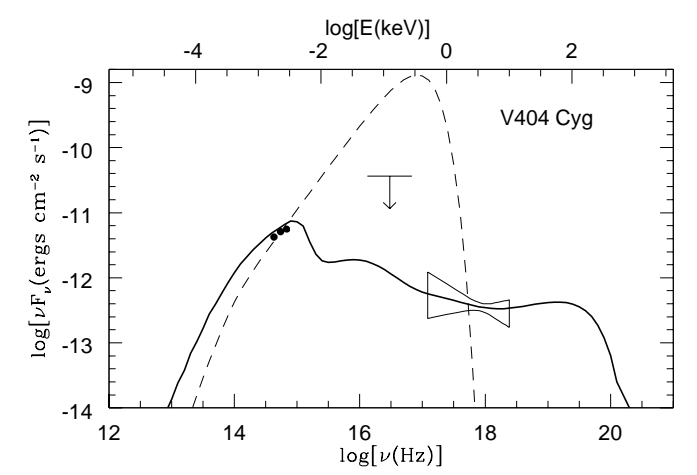

Fig. 2. The spectrum of the BHB source V404 Cyg in quiescence. The three dots on the left represent optical fluxes (Narayan et al. 1996), the error box in the X-ray band corresponds to $2 \sigma$ limits from ASCA (Narayan et al. 1997a), and the upper limit in the EUV is derived from the absence of a HeII $\lambda 4686$ line. The solid line corresponds to an ADAF model with $\dot{M} \sim 0.005 \dot{M}_{\text {Edd }}$. The dashed line is a thin disk model whose $\dot{M}$ has been adjusted to fit the optical data. This model fits poorly in the X-ray band and is inconsistent with the EUV limit. (From Narayan et al. 1997a)

Zdziarski et al. 1996, 1998; Gierlinski et al. 1997).

The situation changed with the recognition of the ADAF solution. This model turned out to have the precise properties - density, electron temperature, stability - needed to explain the hard state. The solid line in Fig. 1 shows an ADAF model of J0422 (Esin et al. 1998) in which the accretion rate has been adjusted to fit the spectrum; the required rate is about a tenth of the Eddington mass accretion rate $\dot{M}_{\text {Edd }}$, where $\dot{M}_{\text {Edd }}=L_{\text {Edd }} /\left(0.1 c^{2}\right)$, i.e., it is the mass accretion rate at which a disk with radiative efficiency 0.1 would radiate at the Eddington luminosity. It is gratifying that both the temperature (which determines the position of the peak) and the Compton $y$-parameter (which determines the power-law slope below the peak) are reproduced well. Esin et al. $(1997,1998,2001)$ present models of other BHBs in the hard state.

A typical accreting $\mathrm{BH}$ observed in the hard state has a luminosity on the order of a few percent of Eddington. At much lower luminosities, we have the quiescent state, where the spectrum becomes noticeably different. Figures 2 and 3 show observations of a quiescent BHB, V404 Cyg, and a quiescent supermassive BH, the Galactic Center source Sgr A*. Although these spectra look very different from the one shown in Fig. 1, the ADAF model is able to fit these and other observations of quiescent systems (Narayan, McClintock \& Yi 1996; Narayan et al. 1997a; Yuan et al. 2003). All it requires is a lower 


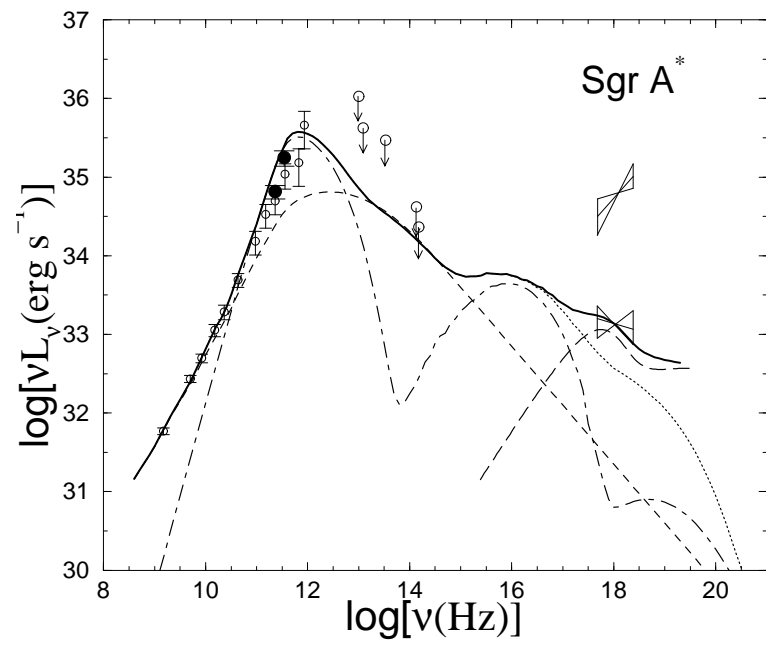

Fig. 3. The quiescent spectrum of Sgr A*. The radio data are from Falcke et al. (1998; open circles) and Zhao et al. (2003; filled circles), the IR data are from Serabyn et al. (1997) and Hornstein et al. (2002), and the two "bow-ties" in the $\mathrm{X}$-ray band correspond to the quiescent (lower) and flaring (higher) data from Baganoff et al. (2001, 2003). The solid line is an ADAF model of Sgr $\mathrm{A}^{*}$ in the quiescent state. The mass accretion rate is $\dot{M} \sim 10^{-6} \dot{M}_{\text {Edd }}$ near the BH. (From Yuan et al. 2003)

value of $\dot{M}$, as appropriate for the lower luminosity. The qualitative features of the spectrum, e.g., a softening of the X-ray power-law index (see Corbel, Tomsick \& Kaaret 2006), follow naturally. However, a caveat is in order: In some cases, the X-ray emission in quiescence may be from a jet lauched from the ADAF ( $\S 3.6)$, rather than from the ADAF itself (e.g., Yuan \& Cui 2005).

\subsection{Radiative Efficiency}

As expressed in equation (4), the defining property of an ADAF is that it is radiatively inefficient,

ADAF : $\quad \eta \equiv L / \dot{M} c^{2} \ll 0.1$.

Calculations show that the ADAF solution is possible only for low mass accretion rates. Specifically, only when $L \lesssim 0.1 L_{\mathrm{Edd}}$ is the gas density low enough to permit a two-temperature plasma (NY95b; Narayan 1996; Esin et al. 1997). Near the critical luminosity $L_{\text {crit }}$ or critical mass accretion rate $\dot{M}_{\text {crit }}$ at which the ADAF solution first becomes viable, the radiative efficiency is, by continuity, not very different from that of a thin disk: $\eta \sim 0.1$. However, with decreasing $\dot{M}$, the efficiency decreases. Very roughly, we estimate (Fig. 4)

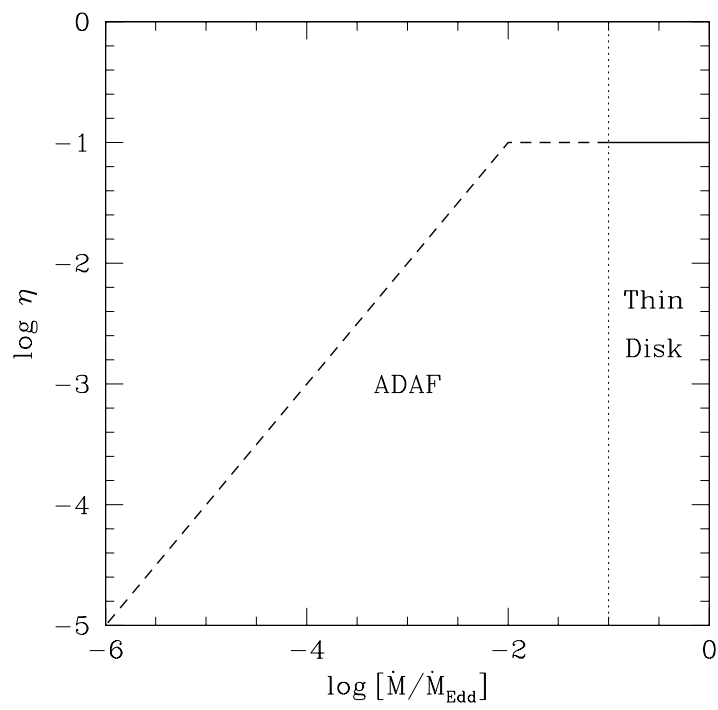

Fig. 4. Radiative efficiency $\eta$ of an accretion flow around a $\mathrm{BH}$, as a function of the Eddington-scaled mass accretion rate (Hopkins, Narayan \& Hernquist 2006b). The horizontal segment between 0.01 and 0.1 of the Eddington accretion rate shows a transition regime in which a part of the accretion flow is an ADAF, but the radiative efficiency is still large. It might correspond to the Intermediate State, and perhaps the upper end of the Hard State (§3.5, Fig. 5). Although this plot is based on calculations shown in Fig. 11 of NY95b and Fig. 13 of Esin et al. (1997), it is still very approximate.

$\eta \sim 0.1\left(\dot{M} / 0.01 \dot{M}_{\text {Edd }}\right), \quad \dot{M}<0.01 \dot{M}_{\text {Edd }}$.

(We note that for the different prescription for $\delta$ used by Sharma et al. 2006, $\eta$ falls rapidly only for $\dot{M} \lesssim 10^{-4} \dot{M}_{\text {Edd. }}$ )

The extreme inefficiency of an ADAF at very low accretion rates is critical for understanding the peculiar properties of accreting BHs in the quiescent state (as first discussed by Narayan et al. 1996 for BHBs and Narayan et al. 1995 for supermassive BHs). We do not discuss this topic here, but point the reader to other reviews for details (e.g., Quataert 2001; Narayan 2002, 2005). The quiescent state has also played a major role in our efforts to test for the presence of an event horizon in BHBs. This is a key topic of $\S 4$.

\subsection{Spectral Regimes}

Based on the properties of the ADAF solution discussed above, Narayan (1996) proposed a simple model for understanding the spectral states of ac- 


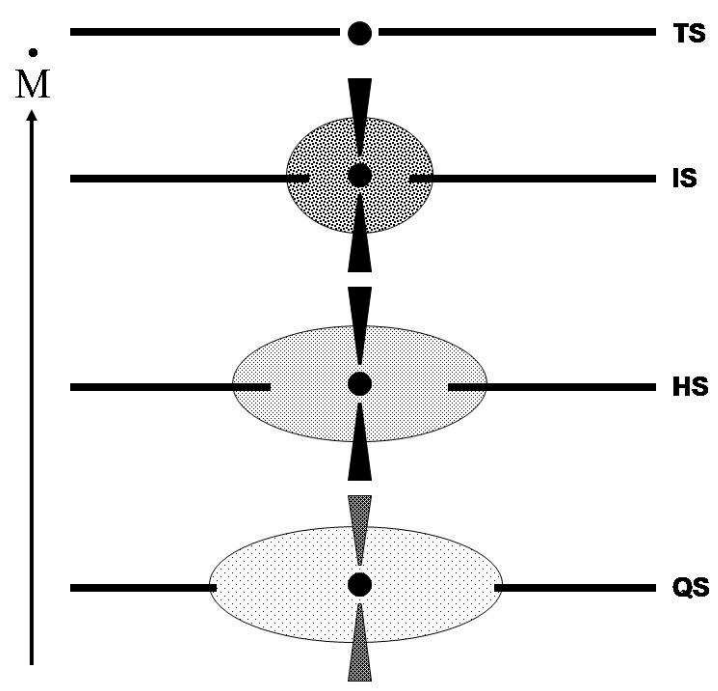

Fig. 5. Configuration of the accretion flow in different spectral states, shown schematically as a function of the mass accretion rate $\dot{M}$ (based on Esin et al. 1997). The ADAF is represented by the hatched ellipses, with the intensity of hatching indicating the density of the hot gas. The horizontal lines represent a standard thin disk. The lowest panel shows the quiescent state (QS), which corresponds to a very low mass accretion rate (say $<10^{-3} \dot{M}_{\text {Edd }}$ ), a weak jet (§3.6), a low radiative efficiency (Fig. 4), and a large transition radius (Fig. 6). The second panel from the bottom shows the hard state (HS), where the mass accretion rate is higher ( $\left.\sim 10^{-3}-10^{-1.5} \dot{M}_{\text {Edd }}\right)$ but still below the critical rate $\dot{M}_{\text {crit }}$, the jet is stronger, the radiative efficiency is somewhat larger, and the transition radius is smaller. The second panel from the top shows the intermediate state (IS), where $\dot{M} \sim 10^{-1.5}-10^{-1} \dot{M}_{\text {Edd }} \lesssim \dot{M}_{\text {crit }}$, the jet is even stronger, the radiative efficiency is high $\sim 0.1$, and the transition radius is fairly close to the ISCO. Finally, the top panel shows the thermal state (TS), where there is no ADAF, the thin disk extends down to the ISCO, $\eta \sim 0.1$, and there is no jet. Esin et al. (1997) included a tentative proposal for the socalled very high state (see also Done et al. 2007), now called the steep power-law state (SPL, McClintock \& Remillard 2006), but we do not include this still-mysterious state here.

creting BHs. This picture was developed in detail by Esin et al. (1997). The basic idea is illustrated in Fig. 5.

The key parameter that determines the spectral state of an accreting BH is $\dot{M}$. When $\dot{M}>\dot{M}_{\text {crit }}$, only the thin disk solution is available, and so the accretion flow is in the form of a thin disk all the way down to the innermost stable circular orbit (ISCO). The system is then in the thermal state, and its spectrum is well-described by the MCD model.

Once $\dot{M}$ falls below $\dot{M}_{\text {crit }}$, both the thin disk and ADAF solutions become viable (at least at small radii). Now accretion continues as a thin disk at radii larger than a transition radius, $R>R_{\mathrm{tr}}$, but the flow switches to an ADAF at smaller radii. When the transition from a pure thin disk (thermal state) to a disk-plus-ADAF configuration first occurs, i.e., when $\dot{M}$ is just below $\dot{M}_{\text {crit }}$, the ADAF is very small in size and we have a more-or-less radiatively efficient flow, with $\eta \sim 0.1$. This corresponds to the so-called intermediate state. Then, at a somewhat lower $\dot{M}$, the ADAF expands a bit and $\eta$ is modestly lower and we have the classic hard state. Finally, when $\dot{M}$ is much lower than $\dot{M}_{\text {crit }}$, the ADAF becomes much larger, $R_{\mathrm{tr}} \sim 10^{3}-10^{4} R_{S}$, with $\eta \ll$ 0.1 (Narayan et al. 1996, 1997a; Menou, Narayan \& Lasota 1999b). This is the quiescent state.

The above paradigm has proved durable (see Zdziarski \& Gierlinski 2004; Done et al. 2007). In particular, considerable evidence has accumulated that the thin disk retreats from the innermost stable circular orbit (ISCO) to a large radius in the quiescent state. The evidence is strongest in transient BHBs and CVs. The spectra of quiescent BHBs show absolutely no sign of any soft blackbodylike X-ray emission from a thin disk at small radii (Narayan et al. 1996, 1997a; McClintock, Narayan \& Rybicki 2004). Timing properties also indicate a large a large size for the ADAF (Hynes et al. 2003; Shahbaz et al. 2005). In additional, theoretical arguments indicate that the thermal-viscous disk instability, which causes the transient behavior in these systems, is incompatible with observations unless the disk is severely truncated in the quiescent state (Lasota, Narayan \& Yi 1996b; Hameury, Lasota \& Dubus 1999; Lasota 2001, 2008; Dubus, Hameury \& Lasota 2001; Yungelson et al. 2006). There are also indications from the time delay between the optical and X-ray outbursts in the BHB GRO J1655-40 (Orosz et al. 1997; Hameury et al. 1997) that the cool disk is truncated at a large radius in the quiescent state.

In the case of supermassive BHs in the quiescent state, there is no feature in the spectrum that might be associated with a thin disk, suggesting that there is no disk at all; examples are Sgr A* (Narayan et al. 1998a; Narayan 2002) and M87 (Di Matteo et al. 2000, 2003). Intermediate luminosity AGN do exhibit optical emission from a disk, but the "big blue bump" is much less pronounced than in highluminosity AGN (Ho 1999); this suggests that the disk is truncated at a radius $\sim 10-100 R_{S}$ and the interior is filled with an ADAF (Gammie, Narayan \& Blandford 1999; Quataert et al. 1999). Incidentally, the thermal-viscous disk instability does not appear 
to operate in AGN disks (Menou \& Quataert 2001; Hameury, Lasota \& Viallet 2007).

In the more luminous hard state, again, there is considerable spectral evidence that the disk is truncated at a transition radius outside the ISCO, and that the inside is filled with an ADAF-like hot flow. The most spectacular example is the BHB XTE J1118+480, for which observations carried out in the hard state had unprecedented spectral coverage. The observations are fit well with an ADAF model, with a transition radius at $\sim 50 R_{S}$ (Esin et al. 2001). The model even fits the complicated timing behavior of the source (Yuan, Cui \& Narayan 2005). It is hard to imagine that the same data could be explained with any model in which a cool disk extends down to the ISCO. Done et al. (2007) review spectral observations of a number of other BHBs where again the data require a truncated disk. Nemmen et al. (2006) show that both the spectrum and the double-peaked Balmer line profile of the LINER source NGC 1097 are consistent with a disk truncated at a few hundred $R_{S}$.

In an interesting study of Cyg X-1, Gilfanov, Churazov \& Revnivtsev (1999; see Cui et al. 1999; Done et al. 2007; for discussions of other sources) found that, as the characteristic frequency in the variability spectrum of the source increases, the power-law tail in the spectral energy distribution steepens and the amplitude of the reflection component in the spectrum increases. This is exactly what one expects when the transition radius between the outer cool disk and the inner hot ADAF varies. With decreasing transition radius, (i) the noise frequency (which is likely related in some fashion to the Keplerian frequency at the transition radius) should increase, (ii) the hot medium should be cooled more effectively by soft photons from the disk, giving a steeper powerlaw tail, and (iii) the solid angle subtended by the cool disk at the ADAF should increase, and there should be a larger reflection component. Zdziarski, Lubinski \& Smith (1999) have noted that a correlation between spectral slope and reflection is commonly seen in both BHBs and AGN.

A few BHBs in the hard state have been found to show a soft blackbody-like component in their spectra (Balucinska-Church et al. 1995; Di Salvo et al. 2001; Miller et al. 2006a,b; Ramadevi \& Seetha 2007; Rykoff et al. 2007). This could be interpreted as evidence that the thin disk extends all the way down to the ISCO4. Some authors have noted that it is

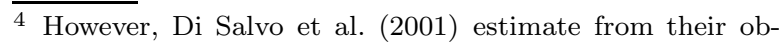

possible for a thin disk to evaporate to an ADAF at a relatively large radius and for the hot gas to then re-condense into a thin disk at small radii (Rozanska \& Czerny 2000; Liu et al. 2007; Mayer \& Pringle $2 0 0 7 \longdiv { 5 }$. Such models might explain the occurrence of a soft spectral component in some hard state sources. However, we note that the soft component typically has only $10 \%$ of the total observed luminosity. It is hard to understand how a radiatively efficient thin disk located at the ISCO could be such a minor contributor to the emitted radiation.

This difficulty is highlighted in the work of D'Angelo et al. (2008). They point out that, in any model of the hard state, there will be considerable interaction between the hot gas which produces the hard X-rays and cool gas that may be present in a conventional disk. The interaction will occur via Xray irradiation (unless one has large outward beaming, which seems unlikely, e.g., Narayan \& McClintock 2005) as well as particle bombardment. Using a prototype model for ion bombardment (Deufel et al. 2002; Spruit \& Deufel 2002; Dullemond \& Spruit 2005), D'Angelo et al. (2008) show that a weak soft component in the X-ray spectrum arises quite naturally when the thin disk is truncated at $\sim 15-20 R_{S} 6$. The model fits the observations surprisingly well; in fact, the same model would predict a much stronger soft component, and would strongly disagree with the observations, if the cool disk were to extend down to the ISCO.

One of the most vexing problems in the theory of ADAFs is that we do not have a robust method of estimating the location of the transition radius $R_{\mathrm{tr}}$. From the earliest studies (e.g., NY95b; Meyer \& Meyer-Hofmeister 1994), it has been plausibly argued that $R_{\mathrm{tr}}$ increases with decreasing $\dot{M}$. However, reliable predictions of the exact dependence have proved difficult, although a number of studies have come up with semi-quantitative results (Meyer \& Meyer-Hofmeister 1994; Honma 1996; Rozanska \& Czerny 2000; Meyer, Liu \& Meyer-Hofmeister 2000; Spruit \& Deufel 2002; Mayer \& Pringle 2007). Yuan

\footnotetext{
servations of $\mathrm{Cyg} \mathrm{X}-1$ that the disk inner edge is located at tens of $R_{S}$, not at the ISCO

5 Models of this kind may give hard spectra without a significant decrease in radiative efficiency. This would be consistent with the transition region between mass accretion rates of 0.01 and 0.1 of Eddington shown in Fig. 4, where we have suggested an ADAF with high radiative efficiency may be present.

6 This is a reasonable location for the transition radius in the hard state (Esin et al. 1998; Done et al. 2007)
} 


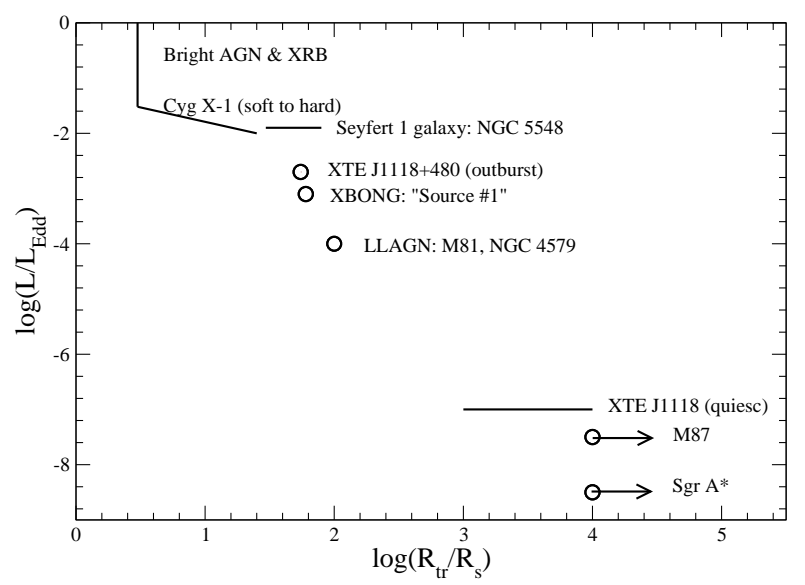

Fig. 6. Estimated location of the dimensionless transition radius $R_{\mathrm{tr}} / R_{S}$, plotted as a function of the Eddington-scaled accretion luminosity $L / L_{\mathrm{Edd}}$, for a sample of BHBs and low-luminosity AGN (Yuan \& Narayan 2004). The individual estimates of $R_{\mathrm{tr}}$ are obtained by fitting spectral observations and are very uncertain. Nevertheless, there seems to be a trend of increasing transition radius with decreasing luminosity.

\& Narayan (2004) tried to use observations to deduce the run of $R_{\mathrm{tr}}$ with accretion luminosity. Figure 6 shows their results.

Another issue that has been discussed recently is hysteresis in the transition between the thermal state and the hard state. Specifically, with increasing $\dot{M}$, the hard state survives up to fairly high luminosities $\sim 0.1-0.2 L_{\mathrm{Edd}}$, whereas with decreasing $\dot{M}$, the transition from the thermal state to the hard state occurs at a much lower luminosity $0.02-0.05 L_{\text {Edd }}$ (Miyamoto et al. 1995; Maccarone \& Coppi 2003; Zdziarski et al. 2004; Done et al. 2007). Meyer-Hofmeister, Liu \& Meyer (2005) have provided a plausible explanation. Their disk evaporation model, coupled with Compton-cooling of the hot electrons in the ADAF, naturally produces a hysteresis in the location of the transition radius.

The brightest systems in the hard state $\left(L \gtrsim 0.1 L_{\text {Edd }}\right)$ are difficult to model with the standard ADAF model. A variant of the ADAF solution - a natural extension of the model - called the luminous hot flow (LHAF; Yuan 2001, 2003; Yuan \& Zdziarski 2004; Yuan et al. 2007; see also Machida, Nakamura \& Matsumoto 2006), looks promising for modeling these sources.

\subsection{Outflows and Jets}

NY94, NY95a discovered an unexpected property of the ADAF solution: the accreting gas in an ADAF has a positive Bernoulli parameter 7 , which is defined as the sum of the kinetic energy, potential energy and enthalpy,

$B e=v^{2} / 2-G M / R+w$.

A positive Bernoulli constant means that the gas is not bound to the BH. (This is not surprising, since the gas is not losing energy through radiation.) Therefore, the above authors suggested that ADAFs should be associated with strong winds and jet:8 (see also Meier 2001).

Strong outflows have been seen in numerical simulations of ADAFs. The first indications came from 2D and 3D hydrodynamic simulations (Stone, Pringle \& Begelman 1999; Igumenshchev \& Abramowicz 2000; Igumenshchev, Abramowicz \& Narayan 2000), but it was soon confirmed in MHD simulations as well (Stone \& Pringle 2001; Hawley \& Balbus 2002; Igumenshchev, Narayan \& Abramowicz 2003; Pen, Matzner \& Wong 2003; Machida, Nakamura \& Matsumoto 2004; McKinney \& Gammie 2004; Igumenshchev 2004).

Apart from producing a gas-dominated, largescale outflow, MHD simulations of ADAFs also have a second distinct outflow component along the axis in the form of a collimated, Poynting-dominated, relativistic jet (McKinney 2005, 2006). The original suggestion of NY94 that ADAFs would have outflows and jets has thus been confirmed by these simulations. Nevertheless, some authors have disputed a connection between a positive Bernoulli parameter and an outflow since it is possible to come up with explicit models that have a positive

\footnotetext{
7 Technically, it should be called the Bernoulli "function" since the quantity varies with radius, but we use Bernoulli "parameter" since that was the term used in NY94

8 There has been a tendency in the literature to ignore these early papers and to credit Blandford \& Begelman (1999) for establishing the connection between ADAFs and outflows. We note that the abstract of the first paper on ADAFs, NY94, states: "Further, the Bernoulli parameter is positive, implying that advection-dominated flows are susceptible to producing outflows...We suggest that advection-dominated accretion may provide an explanation for...the widespread occurrence of outflows and jets in accreting systems." In the second paper, NY95a, the title itself mentions outflows, "Advection-Dominated Accretion: Self-Similarity and Bipolar Outflows," and an entire paragraph of the abstract is devoted to the topic.
} 
Bernoulli parameter but no outflow (Paczyński 1998; Abramowicz et al. 2000).

Observational evidence for the association of nonthermal relativistic jets with ADAFs has accumulated in recent years with the discovery of radio emission in virtually every BHB in the hard state (Corbel et al. 2000; Fender 2001; Fender, Belloni \& Gallo 2004; Fender \& Belloni 2004). The radio emission is generally too bright to be produced by thermal electrons in the accretion flow. It is therefore very likely to come from nonthermal electrons in a jet; in fact, radio VLBI imaging has revealed a resolved jet in a few sources. Thus, it is now observationally well-established that the hard state/ADAF is associated with relativistic jets. According to the discussion in $\S 3.5$, the quiescent state also has an ADAF and should have a (weaker) jet. Indeed, radio emission has been seen from two quiescent systems, V404 Cyg (Hjellming et al. 2000; Gallo, Fender \& Hynes 2005) and A0620-00 (Gallo et al. 2006), confirming this expectation.

How much of the X-ray emission in an ADAF system comes from the accretion flow and how much from the jet? A strong correlation has been seen between radio and X-ray luminosity in the hard state and quiescent state (Corbel et al. 2003; Gallo, Fender \& Pooley 2003). At first sight this might suggest that the X-ray must also come from the jet (e.g., Falcke, Körding \& Markoff 2004). However, since the jet flows out of the ADAF and is thus highly coupled to it, any model in which the X-ray emission is from the ADAF and radio is from the jet is equally compatible with the observations. In recent times, several authors have come down in favor of an ADAF origin for the X-rays (e.g., Heinz \& Sunyaev 2003; Merloni, Heinz \& Di Matteo 2003; Heinz 2004; Heinz et al. 2005; Yuan et al. 2005).

Additional evidence on this issue comes from hard state spectra indicating thermal emission (e.g., see Fig. 1), which is very different from the nonthermal power-law emission one expects from a jet. Markoff, Falcke \& Fender (2001), among others, have argued that the X-ray emission is due to synchrotron emission from a carefully tuned distrubution of nonthermal electrons. However, Zdziarski et al. (2003) and Zdziarski \& Gierlinski (2004) showed that the highenergy cutoff in the spectra of BHBs in the hard state, which arises naturally in a thermal ADAFlike model, is very difficult to reproduce in a nonthermal synchrotron model. A variant of the jet model assigns the X-ray emission to thermal electrons in the "base of the jet" (Markoff, Nowak \&
Wilm 2005), but the discussion then becomes semantic. The base of the jet is surely embedded in the underlying ADAF and it is not clear that one gains anything by simply relabeling the radiation from the ADAF as jet emission. The issue is discussed in greater depth in Narayan (2005), who advocates a 'jet-ADAF' model (as described in Yuan et al. 2005; Malzac, Merloni \& Fabian 2004), in which the high energy X-ray emission in the hard state comes from the ADAF and the low-energy radio (and infrared) emission comes from a jet.

The situation is less clear-cut in the quiescent state, where nonthermal emission from the jet might dominate even in X-rays (Yuan \& Cui 2005; Wu, Yuan \& Cao 2007). It is interesting, however, that the most quiescent system we know, Sgr A*, shows no sign of a jet. Radio images with a resolution of $\sim 15 R_{S}$ appear to be jet-free (Shen et al. 2005), while the quiescent X-ray emission is spatially resolved and appears to be from thermal gas near the Bondi radius at $\sim 10^{5} R_{S}$, not from a jet (Baganoff et al. 2001, 2003).

Turning our attention now to the extended wind that is predicted from an ADAF, and seen in numerical simulations, one consequence of the wind is that, at each radius, a fraction of the accreting gas is lost from the system. Thus, the accretion rate itself varies with radius. This is usually parameterized with an index $s$ such that $\dot{M}(r) \propto r^{s}$. Operationally, this means that, in the self-similar regime, the gas density varies with radius as $\rho \propto r^{-3 / 2+s}$ rather than as $r^{-3 / 2}$ in the NY94 model. Unfortunately, apart from the rather general constraint that $s$ should lie between 0 (no mass loss) and 1 (the limit of a CDAF, §3.7), there is no good theoretical estimate of the value of $s$. We have to resort to numerical simulations or observations. The former tends to give somewhat larger values, e.g., $s \sim 0.7$ (Pen et al. 2003), while the one case where the latter approach has been tried, Sgr A*, yields a smaller value, $s \sim 0.3$ (Yuan et al. 2003).

\subsection{Convection}

Another general property of ADAFs was highlighted by NY94 (see also Begelman \& Meier 1982): ADAFs have entropy increasing inward and should be convectively unstable by the Schwarzschild criterion. Numerical hydrodynamic simulations of ADAFs confirmed the presence of strong convection (Igumenshchev et al. 2000; Narayan, Igumen- 
shchev \& Abramowicz 2000) and led to the development of an analytical self-similar model called the convection-dominated accretion flow (CDAF; Narayan et al. 2000; Quataert \& Gruzinov 2000).

The CDAF model employs a simplified onedimensional treatment of the accretion flow, in which all fluxes are assumed to be in the radial direction. The resulting density varies as $\rho \propto r^{-1 / 2}$. In the language of $\S 3.6$, a CDAF corresponds to $s=1$. However, technically, an idealized CDAF has no mass outflow, only an outward flow of energy by convection; the energy is assumed to flow out into a surrounding medium. In practice, the flow is never perfectly one-dimensional; 2D effects intrude and one expects substantial winds and mass loss as well. A real ADAF thus involves a complicated interplay between convection and winds.

Magnetic fields introduce further complexity, and there has been some discussion in the literature on whether MHD ADAFs do or do not have real convection (Machida, Matsumoto \& Mineshige 2001; Balbus \& Hawley 2002; Narayan et al. 2002b; Pen et al. 2003; Igumenshchev 2004). The question has no practical significance, however, since there is general agreement that ADAFs have both unstable entropy gradients and strong magnetic stresses.

In the particular case of a spherical, non-rotating, MHD flow (the magnetic analog of the Bondi problem), some complications are absent and the problem becomes relatively clean. Here one finds that convection and magnetic fields strongly influence the accretion physics, and the resulting flow is very different from the standard Bondi solution (Igumenshchev \& Narayan 2002; Igumenshchev 2004, 2006). This result is likely to have significant implications for astrophysics; for instance, isolated neutron stars accreting from the interstellar medium will be very much dimmer than one might expect based on the Bondi solution (Perna et al. 2003).

\subsection{ADAFs Around Supermassive Black Holes}

The ADAF solution is essentially independent of the mass $M$ of the central $\mathrm{BH}$. That is, if length and time are scaled by $M$ and the accretion rate is scaled by the Eddington rate (also proportional to $M$ ), then the same solution is valid for any $\mathrm{BH}$ mass. Therefore, any successful application of the ADAF model to a BHB system has immediate consequences for supermassive $\mathrm{BHs}(\mathrm{SMBHs})$ in an equivalent state, and vice versa. This close connection between ADAFs in BHBs and ADAFs in AGN was highlighted in Narayan (1996); it is also empirically obvious from Fig. 6, which combines observations and models of BHBs and AGN.

The first $\mathrm{SMBH}$ to be modeled as an $\mathrm{ADAF}$ was the Galactic Center source Sgr A* (Narayan et al. 1995). This was soon followed by Fabian \& Rees (1995), who suggested that quiescent nuclei in nearby giant ellipticals (e.g., M87) must be accreting via ADAFs, and by Lasota et al. (1996a), who argued that Low Ionization Nuclear Emission-line Region sources (LINERs, e.g., NGC 4258) and lowluminosity active galactic nuclei (LLAGN) must have ADAFs. Both suggestions have turned out to be correct (e.g., Reynolds et al. 1996; Mahadevan 1997; Gammie et al. 1999; Quataert et al. 1999; Di Matteo et al. 2000, 2003; Loewenstein et al. 2001; Ulvestad \& Ho 2001; Nemmen et al. 2006).

Other authors have suggested that all of the following systems have ADAFs: FRI sources (Baum, Zirbel \& O'Dea 1995; Reynolds et al. 1996; Begelman \& Celotti 2004), BL Lac sources (Maraschi \& Tavecchio 2003), X-ray Bright Optically Normal Galaxies (XBONGs; Yuan \& Narayan 2004), and even some Seyferts (Chiang \& Blaes 2003). All of these sources are relatively low-luminosity AGN, where an ADAF is likely to be present (Figs. 5, 6).

As discussed in $\S 3.6$, a feature of the ADAF model is the presence of outflows and jets. This implies that ADAF systems should generally be radio-loud. This is certainly the case for the FRI and BL Lac sources mentioned above. Ho (2002; see also Nagar et al. 2000; Falcke et al. 2000) presents in Fig. 5b of his paper a very interesting plot of radio loudness, defined as the ratio of the flux at $6 \mathrm{~cm}$ to the flux in the optical B band versus Eddington-scaled luminosity, for a sample of galactic nuclei. Virtually every source in the plot with an Eddington-ratio below about 0.01 is radio loud, which is perfectly consistent with our expectation that all such sources should have ADAFs. Moreover, the degree of radio loudness increases with decreasing Eddington ratio, again consistent with our expectation that the ADAF should become more and more dominant with decreasing $\dot{M}$. The majority of sources in Ho's plot that have $L / L_{\text {Edd }}>0.1$ are radio-quiet, as we would expect from Fig. 5 if these systems have cool disks. However, a small minority of these bright sources do show powerful jets (they are generally FRII sources). The exact nature of the accretion in these sources is unclear. A more complete plot, with updated data, can be found in Sikora, Stawarz \& Lasota (2007). 
The jet and the extended wind from an ADAF carry with them substantial kinetic energy. This energy will be dumped into the external medium and will have important consequences. In the context of galaxy formation, there has been discussion recently of the so-called "radio mode" of accretion (Croton et al. 2006) in which outflowing energy from an accreting $\mathrm{SMBH}$ in the galactic nucleus heats up the surrounding medium. This kind of AGN feedback can lead to various effects such as shutting off accretion and/or star formation (Di Matteo et al. 2005; Hopkins et al. 2006a).

It is worth noting that this radio mode is nothing other than the ADAF mode of accretion reviewed in this article. Investigators of AGN feedback may find it profitable to study the considerable work that has been done on ADAFs over the last fifteen years.

\section{The Black Hole Event Horizon}

The first BH, Cygnus X-1, was identified and established in 1972 via a measurement of its mass, which was shown to be too large for a neutron star (NS). The surest evidence for the existence of BHs continues to be through dynamical mass measurements. We now know of 20 additional compact binary X-ray sources (McClintock \& Remillard 2006; Orosz et al. 2007) with primaries that are too massive to be a NS or any stable assembly of cold degenerate matter, assuming that GR is valid. Similarly, dynamical data have established the existence of supermassive BHs, most notably in the nucleus of our Milky Way Galacy (Schödel et al. 2002; Ghez et al. 2005a) and in NGC 4258 (Miyoshi et al. 1995).

Are these compact objects genuine $\mathrm{BHs}$ - pockets of fully collapsed matter that are walled off from sight by self gravity and that, like a shadow, reveal no detail - or are they exotic objects that have no event horizons but manage to masquerade as BHs? Most astrophysicists believe that they are genuine BHs. There are several reasons for this confidence, the most important being that $\mathrm{BHs}$ are an almost inevitable prediction of GR. However, this argument is circular because it presumes that GR is the correct theory of gravity. Furthermore, it ignores the many exotic alternatives to BHs that have been suggested. Thus, the collapsed objects that we refer to throughout as "black holes" are strictly speaking dynamical $\mathrm{BH}$ candidates. The current evidence for BHs is not decisive, nor can dynamical measurements be expected to make it so. We now consider some ap- proaches aimed at establishing that these dynamical $\mathrm{BHs}$ are genuine.

The defining property of a $\mathrm{BH}$ is its event horizon. Demonstrating the existence of this immaterial surface would be the certain way to prove the reality of BHs. Unfortunately, unlike any ordinary astronomical body such as a planet or a star of any kind, it is quite impossible to detect radiation from the event horizon's surface of infinite redshift. (Hawking radiation is negligibly weak for massive astrophysical BHs.) Nevertheless, despite the complete absence of any emitted radiation, it is possible to marshal strong circumstantial evidence for the reality of the event horizon. The fruitful approaches described below are based on comparing X-ray binary systems that contain $\mathrm{BH}$ primaries with very similar systems that contain NS primaries. Such investigations are motivated by the simple fact that the termination of an accretion flow at the hard surface of a NS has observational consequences.

The earliest and strongest evidence for the event horizon is based on the faintness in quiescence of $\mathrm{BH}$ transient systems relative to comparable NS systems. In $\S 4.1$, we discuss the physical arguments that underpin this evidence, and in $\S 4.2$ we present the comparative luminosity data for BHs and NSs. Alternative explanations for the lower luminosities of the $\mathrm{BH}$ systems, which do not involve the event horizon, are considered in $\S 4.3$. In $\S 4.4$, we present three independent and additional arguments for the existence of the event horizon, which are again rooted in comparing BH and NS X-ray binaries. In $\S 4.5$, we discuss the extreme faintness and properties of Sgr $\mathrm{A}^{*}$ and the evidence that this supermassive $\mathrm{BH}$ has an event horizon, and in $\S 4.6$ we argue that even very exotic objects with enormously strong surface gravity (e.g., gravastars) cannot escape our arguments for the event horizon.

\subsection{Accretion and the Event Horizon}

A test particle in a circular orbit at radius $R$ around a mass $M$ has, in the Newtonian limit, kinetic energy per unit mass equal to $G M / 2 R$, and potential energy equal to $-G M / R$. In the context of a gaseous accretion disk, this means that a gas blob at radius $R$ retains $50 \%$ of its potential energy as kinetic energy. The remaining $50 \%$ was transformed into thermal energy during the viscous accretion of the blob to its current radius (Frank et al. 2002).

We now turn to consider accretion on to the ma- 
terial surface of a NS. If the mass $M$ at the center of an accretion disk has a surface, then the kinetic energy in the accreting gas will be converted to thermal energy in a viscous boundary layer and radiated (Frank et al. 2002). In addition, the residual thermal energy that the gas possesses when it reaches the inner edge of the disk will also be radiated from the surface.

If accretion occurs via an ADAF, the luminosity from the accretion disk $L_{\text {acc }}$ and that from the stellar surface $L_{\text {surf }}$ will satisfy

$$
\begin{aligned}
L_{\mathrm{acc}} & \ll G M \dot{M} / 2 R_{*}, \\
L_{\mathrm{surf}} & \approx G M \dot{M} / R_{*} \gg L_{\mathrm{acc}},
\end{aligned}
$$

where $R_{*}$ is the radius of the stellar surface. The accretion luminosity is small because the flow is radiatively inefficient. Therefore, essentially all the potential energy of the accreting gas remains in the gas in the form of thermal and kinetic energy. If the central object has a surface, e.g., it is a NS, the total luminosity we observe will be equal to $L_{\text {surf }}$. However, if the object has an event horizon, i.e., it is a $\mathrm{BH}$, there will be no radiation from a stellar surface and the luminosity we observe will only be equal to $L_{\text {acc. }}$ Thus, we expect

$$
\mathrm{ADAF}: \quad L_{\mathrm{NS}} \gg L_{\mathrm{BH}} \text {. }
$$

Figure 7 shows schematically the luminosity difference we predict between a NS and a $\mathrm{BH}$. This plot is based on the accretion efficiency estimate shown in Fig. 4. Especially at low mass accretion rates, say $\dot{M} \lesssim 10^{-4} \dot{M}_{\text {Edd }}$ (deep quiescent state), we expect a huge luminosity difference between NSs and BHs. Efforts to test this prediction are described in $\S 4.2$.

If the accretion disk is radiatively efficient, then the gas has little thermal energy when it reaches the inner edge of the disk. In this case, we expect 9

$$
\begin{aligned}
L_{\mathrm{acc}} & \approx G M \dot{M} / 2 R_{*}, \\
L_{\mathrm{surf}} & \approx G M \dot{M} / 2 R_{*} \sim L_{\mathrm{acc}} .
\end{aligned}
$$

The above result is based on a Newtonian analysis and is okay so long as the central object has a radius

\footnotetext{
9 The result given here is for a non-spinning accretor. If the central mass is spinning, the boundary layer luminosity from the surface will be somewhat smaller. However, it requires very fine tuning to make $L_{\text {surf }} \ll L_{\text {acc }}$. Moreover, if the central mass spins too close to the "break-up" limit, the extra luminosity due to the surface can actually exceed $G M \dot{M} / 2 R_{*}$ by a large factor (Popham \& Narayan 1991).
}

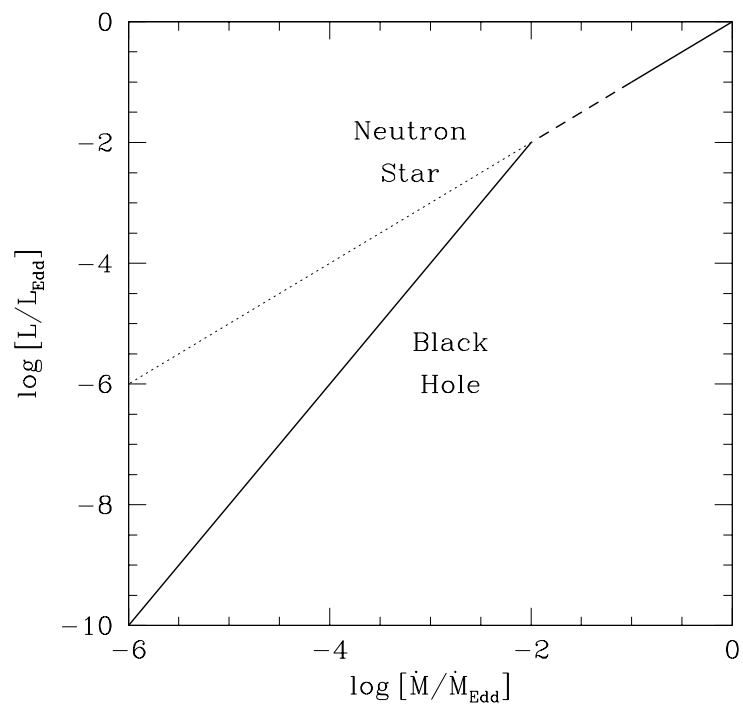

Fig. 7. Predicted luminosities of accreting BHs and NSs as a function of mass accretion rate. This plot is based on the accretion efficiency prescription shown in Fig. 4. Because a NS has a surface, the total luminosity is always $\sim G M_{\mathrm{NS}} \dot{M} / R_{\mathrm{NS}} \sim 0.1 \dot{M} c^{2}$, regardless of whether the accretion flow is radiatively efficient or not. In the case of a $\mathrm{BH}$, however, the radiative efficiency $\eta$ of the accretion flow is of paramount importance, and the observed luminosity becomes $\ll 0.1 \dot{M} c^{2}$ when the accretion rate is highly sub-Eddington.

$R_{*} \gtrsim R_{\mathrm{ISCO}}$, the radius of the ISCO. For more compact objects, we must allow for the fact that, inside the ISCO, the accreting gas no longer spirals in by viscosity but free-falls in the gravitational potential of the central mass. Now we have

$$
\begin{aligned}
L_{\mathrm{acc}} & \approx 0.1 \dot{M} c^{2} \\
L_{\mathrm{surf}} & \approx G M \dot{M} / R_{*}-L_{\mathrm{acc}}>L_{\mathrm{acc}} .
\end{aligned}
$$

The accretion luminosity is limited by the binding energy of the gas at the ISCO, which gives a radiative efficiency $\eta \sim 0.1$. On the other hand, the total energy budget is $G M \dot{M} / R_{*}$, which means that a larger fraction of the luminosity is released at the surface. (The free-falling gas inside the ISCO, crashes on the surface and releases its energy in a shock.) In the limit when $R_{*}$ is arbitrarily close to $R_{S}$ (cf., the discussion of gravastars in $\S 4.6$ ), the total luminosity is equal to $\dot{M} c^{2}$, i.e., the entire rest mass energy of the accreting gas is converted to radiation (100\% radiative efficiency). Note that all luminosities discussed here refer to measurements by 
an observer at infinity.

Combining the results in equations (15), (18) and (20), we see that, regardless of the radius of the accretor and whether the accretion flow is radiatively efficient or inefficient, we expect

Any Accretion Flow: $\quad L_{\text {surf }} \gtrsim L_{\text {acc }}$.

Although this relation is weaker than (15), it can still be used in favorable cases to test for the presence of an event horizon ( $\$ 4.5)$.

\subsection{Evidence for the Event Horizon: Luminosities of Quiescent X-ray Binaries}

$\mathrm{BH}$ and NS transient binary systems are very similar in many respects, and it is reasonable to expect that their mass accretion rates and luminosities would be comparable under similar conditions. There is, however, one important qualitative difference between the two kinds of object — NSs have surfaces whereas BHs do not. Often, this difference is not important. However, as discussed in $\S 4.1$ (see eq. 16), when accretion occurs via an ADAF, a NS binary should be much more luminous than a $\mathrm{BH}$ binary (NY95b). The difference will be especially large in the quiescent state, when the accretion flow is radiatively extremely inefficient (Fig. 7).

Narayan, Garcia \& McClintock (1997b) and Garcia, McClintock \& Narayan (1998) collected available X-ray data on quiescent NS and BH transients and showed that $\mathrm{BH}$ systems are consistently fainter than NS systems. This was the first indication that the objects that astronomers call "black holes" are indeed genuine BHs with event horizons.

Lasota \& Hameury (1998) made the important point that it is necessary to compare quiescent NS and $\mathrm{BH}$ transients at similar mass accretion rates, and the surest way to achieve this is to plot luminosities as a function of the binary orbital period $P_{\text {orb }}$ (the relevant arguments are outlined in Menou et al. 1999b; Lasota 2000, 2008). Since 1999, this has been the standard way of plotting the data (Menou et al. 1999a; Garcia et al. 2001; Narayan, Garcia \& McClintock 2002a; Hameury et al. 2003; McClintock et al. 2004). Figure 8 shows the current status of the comparison, with the luminosities of quiescent $\mathrm{BH}$ and NS transients plotted in (most-appropriate) Eddington units. Fig. 9 shows the same data without the luminosities being scaled. It is clear that, for comparable orbital periods, the $\mathrm{BH}$ systems are 2 to 3 orders of magnitude fainter than their NS cousins.

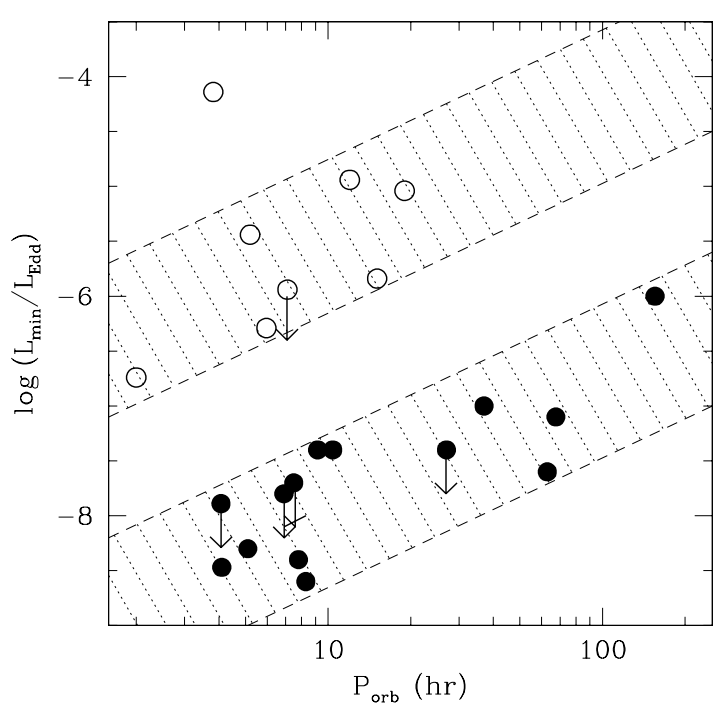

Fig. 8. Eddington-scaled luminosities $(0.5-10 \mathrm{keV})$ of $\mathrm{BH}$ transients (filled circles) and NS transients (open circles) versus the orbital period (see Garcia et al. 2001; McClintock et al. 2004). Only the lowest quiescent detections or Chan$d r a / X M M$ upper limits are shown. The plot shows all systems with known orbital periods, which have optical counterparts and good distance estimates. The diagonal hatched areas delineate the regions occupied by the two classes of sources and indicate the observed dependence of luminosity on orbital period. Note that the BH systems are on average nearly 3 orders of magnitude fainter than the NS systems with similar orbital periods.

As discussed in $\$ 4.2$, a radiatively inefficient ADAF provides a natural explanation for the large luminosity deficit of the BH systems. In this model, the gas approaches the center with a large amount of thermal energy. A BH is dim because the bulk of this thermal energy is trapped in the advective flow, passes through the event horizon, and is lost from sight. On the other hand, a NS is bright because the thermal energy is radiated from its surface.

\subsection{Other Explanations for the Relative Faintness of Quiescent Black Hole Binaries}

A number of alternative models have been put forward in an attempt to rationalize the luminosity differences between quiescent NS and BH systems. Notice below that some of these models generate Xray emission without invoking accretion at all. We first consider a recent challenge centered on the discovery of the extremely low-luminosity NS transient 


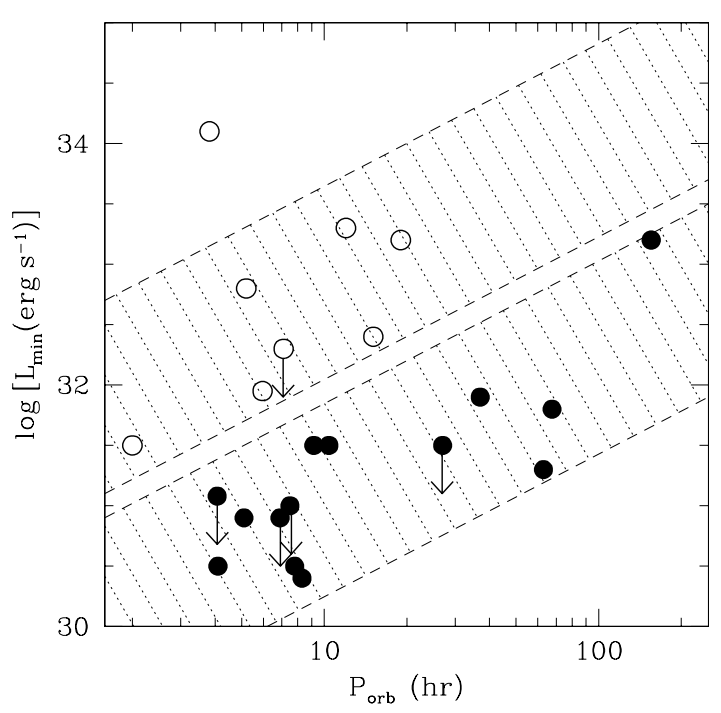

Fig. 9. Same as Fig. 8 except that shown here are the observed luminosities without Eddington scaling. In this representation, the $\mathrm{BH}$ systems are on average a factor of $\sim 100$ fainter than NS systems with similar orbital periods.

$1 \mathrm{H} 1905+00$. Next, we discuss the possibility that the bulk of the accretion power is channeled into a steady jet rather than into X-ray emission. We then consider several diverse models that are discussed in further detail in Narayan et al. (2002a).

The case of $1 \mathrm{H} 1905+00$ : Jonker et al. (2007) claim that the extremely low X-ray luminosity of the NS transient and type I burst source 1H 1905+00 (hereafter, H1905) undermines the evidence summarized in $\S 4.2$ for the existence of the event horizon. The luminosity of this NS $\left(\approx 2 \times 10^{30} \mathrm{erg} \mathrm{s}^{-1} ; D=\right.$ $10 \mathrm{kpc})$, is the same as the luminosity of A0620-00 and several other short-period $\left(P_{\text {orb }} \approx 4-12 \mathrm{hr}\right) \mathrm{BH}$ systems. Based on this result, Jonker et al. assert that the evidence for event horizons is "unproven." However, they ignore a key point of our argument: Namely, as discussed above and illustrated in Figures 8 and 9 , the case for event horizons depends critically on comparing $\mathrm{BH}$ and NS systems with similar orbital periods.

We first note that the orbital period of H1905 is unknown. More importantly, this unknown period is believed to be very short - so short that there are no BH systems with comparable periods. This conclusion is based on deep optical imaging data and the lack of a counterpart. Jonker et al. (2007) conclude that the secondary "can only be a brown or a white dwarf," and that the system is probably an ultracompact binary. Hence the orbital period is likely to be tens of minutes, far less than the shortest $\mathrm{BH}$ binary period of $4.1 \mathrm{hr}$.

Thus, there is no $\mathrm{BH}$ system comparable to H1905. And there is no way to usefully predict the mass accretion rate, as Jonker et al. conclude in the paper's final sentence. The rough trend of luminosity with period indicated in Figure 9 for NSs might imply a very low luminosity for H1905, as observed, but such an extrapolation is unwarranted. See Lasota $(2007,2008)$ for a more detailed discussion of this and other issues.

Jonker et al. (2007) additionally argue that an unknown amount of mass transfered from the secondary might be lost from the system in winds or jets. Their one example of a wind, viz., an extraordinary mass-loss episode observed in GRO J1655-40 (Miller et al. 2006c) when the source was in outburst and had a luminosity $\sim 10^{6}$ times the quiescent level, is quite inappropriate. Also, their mention of possible mass expulsion for quiescent NSs via the propeller mechanism (Lasota \& Hameury 1998; Menou et al. 1999a), if effective, would reduce the luminosities of NSs and would only strengthen our argument.

Jet outflows: Fender et al. (2003) argue that transient BHs at low accretion rates $\left(L / L_{\mathrm{Edd}}<10^{-4}\right)$ "should enter 'jet-dominated' states," in which the majority of the accretion power drives a radiativelyinefficient jet. As we have seen in $\S 3.6$, there is good evidence for this. For instance, the presence of a radio jet has been reasonably well established in quiescence for A0620-00 (Gallo et al. 2006), the closest and one of the least luminous of the BHs in question. Fender et al. (2003) appeal to the empirical result that BHs are $\sim 30$ times as 'radio loud' as NSs at similar accretion rates to deduce that quiescent $\mathrm{BHs}$ should be $\sim 100$ times less luminous in X-rays than quiescent NSs. Their argument is a little convoluted since, if the radio and X-ray emission in quiescence come from the jet (see §3.6), one would think the $\mathrm{BHs}$ would be $\sim 30$ times more luminous, not 100 times less luminous. Therefore, naively, the jet argument only makes the discrepancy in Figs. 8 and 9 more severe. Fender et al. (2003) get around this difficulty by postulating, in addition to different jet efficiencies, also different origins for the $\mathrm{X}$-rays seen in quiescent BHs and NSs.

It is unsatisfying that the reason for the difference in jet activity between BHs and NSs "remains unclear." At bottom, the authors are suggesting that in 
quiescence the BHs are in the 'jet-dominated' regime and the NSs are, "if not jet-dominated, close to the transition to this regime." A key uncertainty in this suggestion is whether the scaling relation that has been established for BHs between radio luminosity and jet luminosity $\left(L_{\text {radio }} \propto L_{\mathrm{x}}^{0.7}\right)$ is also valid for NSs, as is assumed. In addition, Occam's razor suggests that the relative faintness of BHs is unlikely to be attributable to their stronger jets: In $\S 4.5$, we show that Sagittarius $\mathrm{A}^{*}$, which is radiating in quiescence at the same level of Eddington-scaled luminosity as A0620-00, does not possess an energeticallyimportant jet.

Coronal emission from $\mathrm{BH}$ secondaries: The quiescent X-ray luminosity of the $\mathrm{BH}$ systems has been attributed to a rotationally-enhanced stellar corona in the secondary star (Bildsten and Rutledge 2000, but see Lasota 2000). However, as discussed in detail by Narayan et al. (2002a), the luminosities of three of the systems plotted in Figures 8 and 9 exceed by a factor of 6-60 the maximum predicted luminosity of the coronal model; likewise the three systems with adequate data quality show X-ray spectra that are harder/hotter than that typically seen in stellar coronae. Finally, if stellar coronae do contribute at some level, then the accretion luminosities of the $\mathrm{BHs}$ are even lower than our estimates, which would further strengthen the evidence for event horizons.

Incandescent neutron stars: The quiescent luminosity of NS transients has been attributed to heating of the star's crust during outburst followed by cooling in quiescence. This model likewise has problems. (For details and references, see Narayan et al. 2002a.) Briefly, the rapid variability of the prototypical NS transient system Cen X-4 is not expected in a cooling model and implies that no more than about a third of the quiescent luminosity is due to crustal cooling. Furthermore, power-law tails that carry about half the total luminosity are observed for many NS transients (e.g., Cen X-4 and Aql X1). These are unlikely to arise from a cooling NS surface, but could easily be produced by accretion. Finally, strong evidence for continued accretion in quiescence comes from optical variability, which is widely observed for these quiescent systems.

Pulsar wind/shock emission: In another accretionless model, the NS transient switches to a radio pulsar-like mode in quiescence (Campana \& Stella 2000). The X-ray luminosity is expected to be of order the "pulsar shock" luminosity $\sim 2 \times 10^{32} \mathrm{erg} \mathrm{s}^{-1}$, which is close to the observed level. Both the observed power-law and thermal components of emis- sion (see the previous paragraph) are naturally explained by this model: the former component is produced by the shock and the latter is radiated from the NS surface. The lack of any significant periodicity in the quiescent emission (in any electromagnetic band) could be a problem for this model. Millisecond X-ray pulsars such as SAX J1808.4-3658 do show periodicities in outburst, but that emission is clearly the result of accretion, not a pulsar wind/shock.

Optical/UV luminosity: Campana \& Stella (2000) note that the comparison shown in Figs. 8 and 9 assumes the X-ray luminosity is an accurate measure of the accretion rate near the BH or NS. They argue that the optical and UV luminosity also originates near the central object and should therefore be included in the comparison. The non-stellar optical/UV luminosity is much greater than the X-ray luminosity. When it is included, the difference between the BH and NS systems largely disappears. However, as detailed in Narayan et al. (2002a), there are some problems with this argument. The level of optical/UV emission generated in the inner region of ADAFs is strongly suppressed by winds and convection, as evidenced by a comparison of the luminosities of NSs and white dwarfs (Loeb, Narayan \& Raymond 2001). It thus appears unlikely that the optical and UV emission is generated in the hot gas close to the accretor (see Shahbaz et al. 2005). In the case of $\mathrm{CVs}$, it has been established that a large fraction of the optical/UV emission comes from the "hot spot," and it is quite reasonable to expect that this is true for BH and NS systems as well (there is some evidence to support this; Narayan et al. 2002a). Further observations are needed to determine the origin of optical/UV emission in these systems.

\subsection{Further Evidence for the Event Horizon in BHBs}

In addition to the arguments discussed above, we briefly summarize three additional lines of evidence for the existence of event horizons. All three are based on comparisons between BH and NS X-ray binaries. As in the examples above, the first argument considers quiescent systems, whereas the latter two consider active states of accretion.

In quiescence, a soft component of thermal emission is very commonly observed from the surfaces of accreting NSs, which is widely attributed to either deep crustal heating (Brown, Bildsten \& Rutledge 1998) or to accretion. No such component is 
present in the spectrum of the quiescent BHB XTE J1118+480 (hereafter J1118), as one would expect if the compact X-ray source is a bona fide $\mathrm{BH}$ that possesses an event horizon (McClintock et al. 2004). Because of the remarkably low column density to $\mathrm{J} 1118\left(N_{\mathrm{H}} \approx 1.2 \times 10^{20} \mathrm{~cm}^{-2}\right)$ the limit on a hypothetical thermal source is very strong $\left(k T_{\infty}<0.011\right.$ $\mathrm{keV})$; it is in fact a factor of $\sim 25$ lower in flux than the emission predicted by the theory of deep crustal heating, assuming that J1118 has a material surface analogous to that of NSs. Likewise, there is no evidence that accretion is occurring in quiescence onto the surface of J1118, which is the mechanism often invoked to explain the far greater thermal luminosities of NSs. The simplest explanation for the absence of any thermal emission is that J1118 lacks a material surface and possesses an event horizon.

Type I X-ray bursts are very common in NS X-ray binaries, but no type I burst has been seen among the BH systems. A model developed by Narayan \& Heyl (2002, 2003), which reproduces the gross observational trends of bursts in NS systems, shows that, if the dynamical BHs have surfaces, they should exhibit instabilities similar to those that lead to type I bursts on NSs. Remillard et al. (2006), following earlier work by Tournear et al. (2003), measured the rates of type I X-ray bursts from a sample of 37 nonpulsing X-ray transients observed with $R X T E$ during 1996-2004. Among the NS sources, they found 135 type I bursts in 3.7 Ms of PCA exposures (13 sources) with a burst rate function consistent with the Narayan \& Heyl model. However, for the BH group (18 sources), they found no confirmed type I bursts in $6.5 \mathrm{Ms}$ of exposure. Their upper limit on the incidence of burst activity in these sources is inconsistent with the model predictions at a high statistical significance if the accretors in BHBs have solid surfaces. The results provide strong indirect evidence for $\mathrm{BH}$ event horizons, and it would appear that the evidence can be refuted only by invoking rather exotic physics.

Likewise drawing on the extensive archive of RXTE data, Done and Gierlinski (2003) have examined the patterns of X-ray spectral evolution of active $\mathrm{BH}$ and $\mathrm{NS}$ sources and identified a distinct type of soft spectrum that is occasionally observed only in the $\mathrm{BH}$ sources. They attribute this spectrum to thermal emission from the inner accretion disk (corresponding to the high state discussed earlier). They then argue that NSs with a similar accretion rate cannot exhibit such a simple, low-temperature spectrum because they would have a second component in the emission from the boundary layer where accreting matter impacts the stellar surface. They present a thermal/nonthermal Comptonization model for the boundary layer emission which has significant uncertainty, given that so many details of the accretion physics are complex and poorly understood. Nevertheless, Done \& Gierlinski (2003) do appear to have identified a systematic difference in the X-ray spectra of accreting BHs and NSs, and it is surely worth pursuing this signature in the effort to amass evidence for the reality of event horizons.

\subsection{Event Horizon in Sagittarius $A^{*}$}

The supermassive $\mathrm{BH}$ in $\mathrm{Sgr} \mathrm{A}^{*}$ has a mass $M \sim 4 \times 10^{6} M_{\odot}$ (Schödel et al. 2002; Ghez et al. 2005a) but an accretion luminosity of only $\sim 10^{36} \mathrm{erg} \mathrm{s}^{-1}$; thus the source is highly subEddington, $L \lesssim 10^{-8} L_{\text {Edd }}$. From our earlier discussion, the accretion flow must be in the form of an ADAF, i.e., the radiating gas must be extremely hot and optically thin. This expectation is confirmed by the $10^{10} \mathrm{~K}$ brightness temperature of the radio/millimeter emission (Shen et al. 2005) and the fact that most of the emission is in this band rather than at frequencies $\nu \sim k T / h$ (X-ray $/ \gamma$-ray band).

The ADAF model has been successfully applied to Sgr A* (Narayan et al. 1995, 1998a; Manmoto et al. 1997; Mahadevan 1998; Yuan et al. 2003; to mention a few). If we assume that the accretion flow is radiatively very inefficient, then it is easy to make a strong case for Sgr A* not having a surface (Narayan et al. 1998a). However, as Broderick \& Narayan (2006, 2007) showed, we can argue for the presence of an event horizon even without assuming an ADAF.

If Sgr $A^{*}$ does not have an event horizon, but has a surface, then any emission from the surface will be blackbody-like. This is because we expect the surface to be optically thick. However, the radio/millimeter emission mentioned above cannot be from this surface because of its incredibly high $10^{10} \mathrm{~K}$ brightness temperature. Thus, the radio/millimeter radiation is from the accretion flow, and its luminosity gives a strict lower bound on $L_{\text {acc }}$. By equation (21) then, we expect a luminosity of at least $L_{\text {surf }} \sim 10^{36} \mathrm{erg} \mathrm{s}^{-1}$ from the surface. The surface radiation would be thermal and blackbodylike and should come out in the infrared (as easily shown, given the luminosity and the likely area of the surface). However, there is no sign of it! 


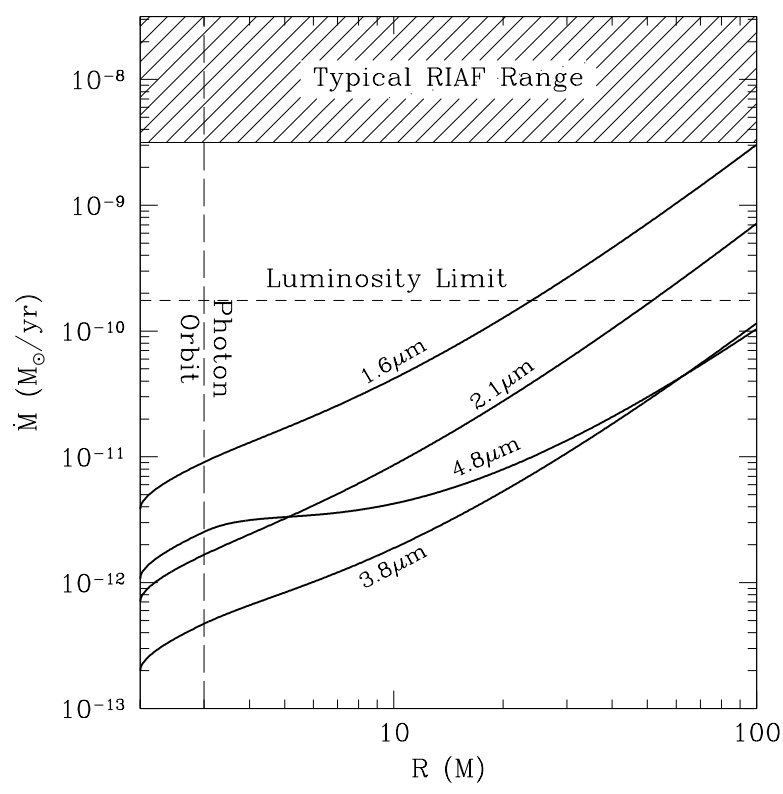

Fig. 10. The four solid lines show independent upper limits on the mass accretion rate at the surface of Sgr $\mathrm{A}^{*}$ (assuming the source has a surface) as a function of the surface radius $R$. Each limiting curve is derived from a limit on the quiescent flux of Sgr $\mathrm{A}^{*}$ in an infrared band. The hatched area at the top labeled "Typical RIAF Range" corresponds to the mass accretion rate in typical ADAF models of Sgr A* (e.g., Yuan et al. 2003). The horizontal dashed line represents the minimum accretion rate needed to power the bolometric luminosity of Sgr A*. (From Broderick \& Narayan 2006)

Figure 10 shows constraints on the mass accretion rate in Sgr A*. The horizontal dashed line is the minimum accretion rate $\dot{M}_{\text {acc }}$ in Sgr A*; this is the rate needed, even with a radiatively efficient flow $(\eta \sim$ 0.1 ), just to power the observed radio/millimeter radiation. The four solid lines show the maximum mass accretion rate $\dot{M}_{\text {surf }}$ allowed if Sgr $\mathrm{A}^{*}$ has a spherical surface of radius $R$. Each curve corresponds to a measured limit on the steady quiescent infrared flux in a particular band (Stolovy et al. 2003; Clenet et al. 2004; Ghez et al. 2005b) and provides an independent upper limit on $\dot{M}_{\text {surf }}$. For radii less than $15 G M / c^{2}$ (the upper limit on the size of Sgr A*; Shen et al. 2005), we see that all four bands give upper limits on $\dot{M}_{\text {surf }}$ that are far below the minimum $\dot{M}_{\text {acc}}$; in fact, the discrepancy is larger than a factor of 100 in the $3.8 \mu \mathrm{m}$ band. Note that the discrepancy would be much larger if we assumed that the accretion flow is radiatively inefficient (corresponding to the hatched region in Fig. 10).

The only way to avoid the large discrepancy illustrated in Fig. 10 is to give up the assumption that
Sgr A* has a surface. Obviously, if the source has an event horizon, then we do not expect any surface radiation, and there is no problem.

What if Sgr $\mathrm{A}^{*}$ ejects all the accreting mass via a jet before the gas reaches the surface? Is this a viable explanation for the lack of surface emission? We can easily rule out this possibility. Recall that the source of energy in an accretion system is gravity. At a bare minimum, we know that matter is being accreted at a rate $\dot{M}_{\text {acc }}$ (shown by the horizontal dashed line in Fig. 10) in order to produce the observed radiation. All of this mass has to fall into the potential well in order to release its energy. If Sgr $\mathrm{A}^{*}$ has a jet with a kinetic luminosity $L_{\text {jet }}$, the energy for this must also come from accretion. We will then require an even larger $\dot{M}_{\text {acc }}$, and hence a larger $\dot{M}_{\text {surf }}$, since the accreting gas now has to power both the radiation and the jet. The discrepancy with the observed limits on the quiescent infrared flux would then be even larger.

\subsection{Effects of Very Strong Gravity}

The arguments for the event horizon presented in $\S \S 4.2,4.5$ are based on Newtonian ideas. Some authors (e.g., Abramowicz, Kluzniak \& Lasota 2002) have questioned whether the arguments might be substantially modified by strong gravity in the vicinity of the compact accretor.

Buchdahl (1959) showed, for a wide class of reasonable equations of state, that the smallest radius allowed for a compact non-rotating object is $R_{\min }=$ $(9 / 8) R_{S}$. An object with this limiting radius has a gravitational redshift from its surface $z_{\mathrm{gr}}=2$. At such modest redshifts, we do not expect the effects of strong gravity to be particularly large, and so the arguments presented in $\S \S 4.2,4.5$ will continue to hold.

Recently, however, a new class of solutions has been discussed, which goes variously under the name of "gravastar" and "dark energy star" (Mazur \& Mottola 2001; Chapline et al. 2003; Visser \& Wiltshire 2004; Carter 2005; Lobo 2006). In this model, the radius $R$ of an object of mass $M$ is allowed to be arbitrarily close to $R_{S}: \Delta R \equiv R-R_{S} \ll R_{S}$. The surface redshift can then be arbitrarily large:

$1+z_{\mathrm{gr}} \approx\left(R_{S} / \Delta R\right)^{1 / 2} \gg 1$.

Although the gravastar model is very artificia 10 , it is nevertheless interesting to ask whether such a

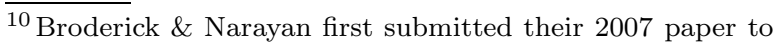


model, which has no event horizon, can explain the observations described in $\S \S 4.2,4.5$ (Abramowicz et al. 2002). Broderick \& Narayan (2006, 2007) show that it cannot. We briefly summarize the arguments here.

A large value of $z_{\mathrm{gr}}$ means that any radiation emitted from the surface is redshifted greatly before it reaches the observer at infinity. This looks like an easy way of hiding the surface luminosity. However, a simple energy conservation argument shows otherwise. Assuming steady state, in the limit as $\Delta R / R_{S} \rightarrow 0$, the total luminosity observed at infinity, $L_{\text {acc }}+L_{\text {surf }}$, must be equal to $\dot{M} c^{2}$ (the rate of accretion of rest mass energy). Moreover, the effective radius of the source as viewed by a distant observer is $(3 \sqrt{3} / 2) R_{S}=2.6 R_{S}$, and so the surface radiation will be in the X-ray band for accreting BHBs (§4.2) and in the infrared for Sgr A* (§4.5), exactly as in the Newtonian analysis.

Could the large gravitational redshift cause a large delay in the signals from the surface of the star, and could this be why we do not see the radiation? The extra delay due to relativity is easily estimated by considering null geodesics in the Schwarzschild metric:

$\Delta t=\left(R_{S} / c\right) \ln \left(R_{S} / \Delta R\right)$.

Since the dependence is only logarithmic, the extra delay is not significant. For instance, even if we take $\Delta R$ to be comparable to the Planck length $\sim$ $10^{-33} \mathrm{~cm}$ (the smallest length we can legitimately consider), the delay is only $\sim 10 \mathrm{~ms}$ for a BHB and $\sim 1000 \mathrm{~s}$ for Sgr A*.

We assumed that the radiation from the surface would have a blackbody-like spectrum. Is this likely? Actually, it is virtually guaranteed as $\Delta R / R_{S} \rightarrow 0$. When the radius of an object is very close to the Schwarzschild radius, most rays emitted from the surface are bent back on to the surface, and only a tiny fraction of rays escapes to infinity. The solid angle corresponding to escape is $\Omega_{\text {esc }} \sim \Delta R / R_{S} \ll 1$. In this limit, the surface behaves just like a furnace with a pinhole (the textbook example of a blackbody!), and so the escaping radiation is certain to have a nearly perfect blackbody spectrum.

Phys. Rev. Lett., but the paper was not even sent out for review because, in the words of the editor, "the gravastar model of Mazur and Mottola is not considered by the community to be a viable alternative." B\&N then submitted the paper to Class. Quantum Grav., where most papers on gravastars and dark energy stars are published.
Could the surface luminosity escape in the form of particles rather than radiation? By the blackbody argument given above, whatever escapes must be in thermodynamic equilibrium at the temperature $T_{\infty}$ observed at infinity. For the sources of interest to us, $k T_{\infty} \sim \mathrm{eV}\left(\operatorname{Sgr} \mathrm{A}^{*}\right)$ and $\mathrm{keV}(\mathrm{BHBs})$. In thermodynamic equilibrium, the only particles with any significant number density at these temperatures are neutrinos. Even if we include all three species of neutrinos, the radiation flux is reduced by a factor of only 8/29 (Broderick \& Narayan 2007). This is a small correction compared to the large discrepancies $\gtrsim 100$ that we described in $\S \S 4.2,4.5$.

Finally, we note that our arguments for the event horizon are based on a steady state assumption. Specifically, we assume that the surface luminosity is proportional to the average mass accretion rate on the surface. In the gravastar model, in particular, one can imagine scenarios in which steady state is not reached. However, Broderick \& Narayan (2007) show that this explanation can be ruled out, at least with current gravastar models.

We thus conclude that strong gravity effects are unable to weaken our arguments for the presence of an event horizon in quiescent BHBs and in Sgr $\mathrm{A}^{*}$. We must look to more conventional astrophysical explanations. The only idea that we find somewhat plausible is that the radiation we observe from quiescent X-ray binaries and Sgr $\mathrm{A}^{*}$ is not produced by accretion at all, but by some other process. This would undercut all our arguments since we assume, as an article of faith, that the radiation we observe is powered by gravity through accretion. Given the success of the accretion paradigm in explaining a vast body of observations on a variety of $\mathrm{BH}$ systems in many different spectral states, it seems rather extreme to abandon the idea of accretion in just those particular sources where we find evidence for the presence of an event horizon.

\section{Conclusion}

The aim of this article is two-fold: To give an updated and current account of the ADAF model, with an emphasis on applications to accreting BHs (§3), and to review the considerable body of evidence presently available for the presence of event horizons in astrophysical BHs (§4).

Observations of $\mathrm{BH}$ binaries $(\mathrm{BHBs})$ and active galactic nuclei (AGN) indicate that, at luminosities of a few percent or less of Eddington, accretion oc- 
curs via a very different mode than the standard thin accretion disk. At these luminosities, sources have hard X-ray spectra, quite unlike the soft blackbodylike spectra seen in more luminous sources. Prior to the establishment of the ADAF model, observations in the hard state were modeled in an ad hoc way using empirical thermal Comptonization models. In the mid-1990s, the ADAF solution was shown to have precisely the densities, temperatures, radiative (in)efficiencies - and stability - required to provide a physical description of the observations. Moreover, since the ADAF model is essentially massindependent, observations of BHBs and AGN are both explained with more-or-less the same ADAF model. The model gives satisfactory results for a wide range of luminosity, from about $10^{-1}$ down to $10^{-8}$ of Eddington (below which there are no observations).

The defining characteristic of an ADAF is that the gas is not radiatively efficient. A significant fraction of the energy released by viscous dissipation is retained in the accreting gas and advected with the flow. The trapped thermal energy causes the accreting gas to be weakly bound to the BH. Based on this property, it was predicted already in the earliest ADAF papers that sources in the ADAF state would have strong winds and jets. Nonthermal radio emission has been detected in recent years from BHBs in the hard state and quiescent state, as well as from all AGN with luminosities below 1\% of Eddington. These sources have spectra consistent with the ADAF model, thus confirming a strong connection between ADAFs and jets/outflows. A currently active topic of research is the role of AGN feedback on galaxy formation. Research in this area can now draw on the extensive literature on ADAFs.

Of special interest in this work are those accreting objects for which there exist estimates or constraints on the two system specific parameters: $\mathrm{BH}$ mass $M$ and mass accretion rate $\dot{M}$. Specifically, we are referring here to Saggitarius $A^{*}$ and a selected sample of quiescent BH and NS X-ray binaries. We have featured these two examples because they provide strong observational evidence for the existence of the event horizon.

In the case of the binaries, one can make a plausible argument that, for comparable orbital periods, the mass accretion rates and luminosities of both types of systems should be comparable. Remarkably, however, the BH systems are observed to be dimmer by a factor of $\sim 100-1000$. We review a wide range of unsatisfactory attempts to explain this large lu- minosity difference. In contrast, the ADAF model provides an entirely straightforward explanation for the faintness of quiescent BHs: A NS must radiate the trapped thermal radiation that is advected with the accretion flow and rains down on its surface, whereas the $\mathrm{BH}$ hides the energy behind its event horizon. Additional evidence for the event horizon is provided in further comparisons of NSs and BHs in outburst: The BHs lack type I X-ray bursts, and they lack a distinctive boundary-layer component of emission. Both of these properties are expected if the BHs possess event horizons, but hard to explain otherwise.

The supermassive $\mathrm{BH}$ in $\mathrm{Sgr} \mathrm{A}^{*}$ is extraordinarily quiescent. The observations strongly support the existence of an ADAF, and it is consequently easy to provide a compelling argument for an event horizon. However, for Sgr $\mathrm{A}^{*}$, one can make an even stronger argument for the lack of a hypothetical material surface without at all invoking an ADAF. The large radio/millimeter accretion luminosity of Sgr $\mathrm{A}^{*}$, which has a brightness temperature $>10^{10} \mathrm{~K}$, obviously cannot be emitted by an optically-thick surface, and so it must be radiated from the accretion flow. This establishes a hard lower limit on the accretion luminosity and $\dot{M}$. Meanwhile, high angular resolution radio observations constrain the radius of the surface to be $<15 R_{\mathrm{S}}$. This constraint and the lower limit on $\dot{M}$ predict a near-IR flux of thermal, blackbody-like surface emission that is far above the observed limits. The obvious explanation is that there is no material surface, only an event horizon. As an added bonus, it is energetically impossible to explain away the lack of surface emission by appealing to mass loss in a jet.

The case for event horizons in both $\operatorname{Sgr} \mathrm{A}^{*}$ and in the stellar-mass BHs is robust against appeals to strong gravity or the leading models of exotic stars. First, GR effects will be mild for nearly all conventional models of degenerate stars, whose radii are restricted to be $\geq 9 / 8 R_{\mathrm{S}}$ and whose surface redshifts are therefore $\leq 2$. Secondly, even for an exotic star (a gravastar) with an extraordinary surface redshift of a million or more, the full accretion luminosity from its surface will be delivered as X-rays (in binaries) or infrared (in Sgr $A^{*}$ ) to a distant observer. Thus, extreme redshifts have practically no effect on the argument for the event horizon.

To play on Carl Sagan's famous comment, there will always be an absence of direct evidence for the event horizon, but this surely cannot be taken as evidence of its absence in nature. On the contrary, 
and with a pun in mind, many indicators show that the event horizon is an inescapable reality.

The authors thank Bozena Czerny, Jean-Pierre Lasota and Feng Yuan for helpful comments.

\section{References}

Abramowicz, M. A., Chen, X., Granath, M., \& Lasota, J. P. 1996, ApJ, 471, 762

Abramowicz, M. A., Chen, X., Kato, S., Lasota, J. P., \& Regev, O. 1995, ApJ, 438, L37

Abramowicz, M. A., Czerny, B., Lasota, J. P., \& Szuszkiewicz, E. 1988, ApJ, 332, 646

Abramowicz, M. A., Kluzniak, W., \& Lasota, J. P. 2002, A\&A, 396, L31

Abramowicz, M. A., Lasota, J. P., \& Igumenshchev, I. V. 2000, MNRAS, 314, 775

Arnaud, K. A. 1996, in ASP Conf. Ser. 191, Astronomical Data Analysis and Systems V, ed. G. H. Jacoby \& J. Barnes (San Francisco: ASP) p17

Baganoff, F. K., et al. 2001, Nature, 413, 45

Baganoff, F. K., et al. 2003, ApJ, 591, 891

Balbus, S. A., \& Hawley, J. F. 2002, ApJ, 573, 749

Balucinska-Church, M., Belloni, T., Church, M. J., \& Hasinger, G. 1995, A\&A, 302, L5

Baum, S. A., Zirbel, E. L., \& O'Dea, C. P. 1995, ApJ, 451, 88

Begelman, M. C. 1979, MNRAS, 187, 237

Begelman, M. C., \& Celotti, A. 2004, MNRAS, 352, L45

Begelman, M. C., \& Chiueh, T. 1988, ApJ, 332, 872

Begelman, M. C., \& Meier, D. L. 1982, ApJ, 253, 873

Bildsten, L., \& Rutledge, R. E. 2000, ApJ, 541, 908

Bisnovatyi-Kogan, G., \& Lovelace, R. V. E. 1997, ApJ, 486, L43

Blackman, E. G. 1999, MNRAS, 302, 723

Blandford, R. D., \& Begelman, M. C. 1999, MNRAS, 303, L1

Broderick, A. E., \& Narayan, R. 2006, ApJ, 638, L21

Broderick, A. E., \& Narayan, R. 2007, Class. Quantum Grav., 24, 659

Brown, E. F., Bildsten, L., \& Rutledge, R. E. 1998, ApJ, 504, L95

Buchdahl, H. A. 1959, Phys. Rev., 116, 1027

Campana, S., \& Stella, L. 2000, ApJ, 541, 849

Carter, B. M. N. 2005, Class. Quantum Grav., 22, 4551

Chapline, G., et al. 2003, Int. J. Mod. Phys. A, 18, 3587
Chen, X., Abramowicz, M. A., \& Lasota, J. P. 1997, ApJ, 476, 61

Chen, X., Abramowicz, M. A., Lasota, J. P., Narayan, R., \& Yi, I. 1995, ApJ, 443, L61

Chiang, J., \& Blaes, O. 2003, ApJ, 586, 97

Clenet, Y., et al. 2004, A\&A, 424, L21

Corbel, S., et al. 2000, A\&A, 359, 251

Corbel, S., Nowak, M. A., Fender, R. P., Tzioumis, A. K., \& Markoff, S. 2003, A\&A, 400, 1007

Corbel, S., Tomsick, J. A., \& Kaaret, P. 2006, ApJ, 636,971

Croton, D. J., et al. 2006, MNRAS, 365, 11

Cui, W., Zhang, S. N., Chen, W., \& Morgan, E. H. 1999, ApJ, 512, L43

D’Angelo, C., Giannios, D., Dullemond, C., \& Spruit, H. 2008, A\&A, submitted

Davis, S. W., Done, C., \& Blaes, O. M. 2006, ApJ, 647,525

Davis, S. W., \& Hubeny, I. 2006, ApJS, 164, 530

Deufel, B., Dullemond, C. P., \& Spruit, H. C. 2002, A\&A, 387, 907

Di Matteo, T., Allen, S. W., Fabian, A. C., Wilson, A. S., \& Young, A. J. 2003, ApJ, 582, 133

Di Matteo, T., Quataert, E., Allen, S. W., Fabian, A. C., \& Narayan, R. 2000, MNRAS, 311, 507

Di Matteo, T., Springel, V., \& Hernquist, L. 2005, Nature, 433, 604

Di Salvo, T., Done, C., Zycki, P. T., Burderi, L., \& Robba, N. R. 2001, ApJ, 547, 1024

Done, C., \& Gierlinski, M. 2003, MNRAS, 342, 1041

Done, C., Gierlinski, M., \& Kubota, A. 2007, A\&AR, 15,1

Dubus, G., Hameury, J. M., \& Lasota, J. P. 2001, A\&A, 373, 251

Dullemond, C. P., \& Spruit, H. C. 2005, A\&A, 434, 415

Esin, A. A., McClintock, J. E., \& Narayan, R. 1997, ApJ, 489, 865

Esin, A. A., Narayan, R., Cui, W., Grove, J. E., \& Zhang, S. N. 1998, ApJ, 505, 854

Esin, A. A., et al. 2001, ApJ, 555, 483

Fabian, A. C., \& Rees, M. J. 1995, MNRAS, 277, L5

Falcke, H., Goss, W. M., Matsuo, H., Teuben, P., Zhao, J. H., \& Zylka, R. 1998, ApJ, 499, 731

Falcke, H., Nagar, N. M., Wilson, A. S., \& Ulvestad, J. S. 2000, ApJ, 542, 197

Fender, R. P. 2001, MNRAS, 322, 31

Fender, R. P., \& Belloni, T. 2004, ARAA, 42, 317

Fender, R. P., Belloni, T. M., \& Gallo, E. 2004, MNRAS, 355, 1105

Fender, R. P., Gallo, E., \& Jonker, P. G. 2003, MNRAS, 343, L99 
Frank, J., King, A., \& Raine, D. J. 2002, Accretion Power in Astrophysics (Cambridge Univ. Press)

Gallo, E., Fender, R. P., \& Hynes, R. I. 2005, MNRAS, 356, 1017

Gallo, E., Fender, R. P., \& Pooley, G. G. 2003, MNRAS, 344, 60

Gallo, E., et al. 2006, MNRAS, 370, 1351

Gammie, C. F., Narayan, R., \& Blandford, R. 1999, ApJ, 516, 177

Garcia, M. R., McClintock, J. E., \& Narayan, R. 1998, ASP Conf. Series, Vol. 137, ed. S. Howell, E. Kuulkers \& C. Woodward, p.506

Garcia, M. R., McClintock, J. E., Narayan, R., Callanan, P., Barret, D., \& Murray, S. S. 2001, ApJ, 553, L47

Ghez, A. M., et al. 2005a, ApJ, 620, 744

Ghez, A. M., et al. 2005b, ApJ, 635, 1087

Gierlinski, M., et al. 1997, MNRAS, 288, 958

Gilfanov, M., Churazov, E., \& Revnivtsev, M. 1999, A\&A, 352, 182

Goldston, J. E., Quataert, E., \& Igumenshchev, I. V. 2005, ApJ, 621, 785

Hameury, J. M., Lasota, J. P., \& Dubus, G. 1999, MNRAS, 303, 39

Hameury, J. M., Lasota, J. P., McClintock, J. E., \& Narayan, R. 1997, ApJ, 489, 234

Hameury, J. M., et al. 2003, A\&A, 399, 631

Hameury, J. M., Lasota, J. P., \& Viallet, M. 2007, in Black Holes from Stars to Galaxies, ed. V. Karas \& G. Matt, Cambridge Univ. Press, p297

Hawley, J. F., \& Balbus, S. A. 2002, ApJ, 573, 738

Hawley, J. F., Gammie, C. F., \& Balbus, S. A. 1996, ApJ, 464, 690

Heinz, S. 2004, 355, 835

Heinz, S., Merloni, A., Di Matteo, T., \& Sunyaev, R. 2005, Astrophys. Sp. Sci., 300, 15

Heins, S., \& Sunyaev, R. 2003, MNRAS, 343, L59

Hjellming, R. M., Rupen, M. P., Mioduszewski, A. J., \& Narayan, R. 2000, ATel, \#54

Ho, L. C. 1999, ApJ, 516, 672

Ho, L. C. 2002, ApJ, 564, 120

Honma, F. 1996, PASJ, 48, 77

Hopkins, P. F., et al. 2006a, ApJS, 163, 1

Hopkins, P. F., Narayan, R., \& Hernquist, L. 2006b, ApJ, 643, 641

Hornstein, S. D., Ghez, A. M., Tanner, A., Morris, M., Becklin, E. E., \& Wizinowich, P. 2002, ApJ, 577,738

Hynes, R. I., Charles, P. A., Casares, J., Haswell, C. A., Zurita, C., \& Shahbaz, T. 2003, MNRAS, 340, 447

Ichimaru, S. 1977, ApJ, 214, 840
Igumenshchev, I. V. 2004, Prog. Theor. Phys. Suppl., 155, 87

Igumenshchev, I. V. 2006, ApJ, 649, 361

Igumenshchev, I. V., \& Abramowicz, M. A. 2000, ApJS, 130, 463

Igumenshchev, I. V., Abramowicz, M. A., \& Narayan, R. 2000, ApJ, 537, L27

Igumenshchev, I. V., \& Narayan, R. 2002, ApJ, 566, 137

Igumenshchev, I. V., Narayan, R., \& Abramowicz, M. A. 2003, ApJ, 592, 2042

Jonker, P. G., Steeghs, D., Chakrabarty, D., \& Juett, A. M. 2007, ApJ, 665, L147

Kato, S., Fukue, J., \& Mineshige, S. 1998, Black Hole Accretion Disks (Kyoto Univ. Press)

Kato, S., Yamasaki, T., Abramowicz, M. A., \& Chen, X. 1997, PASJ, 49, 221

Lasota, J. P. 1996, in Physics of Accretion Disks, ed. S. Kato, J. Fukue \& S. Mineshige (Gordon \& Breach) p85

Lasota, J. P. 1999a, Phys. Rep., 311, 247

Lasota, J. P. 1999b, Sci. Am., 280, 30

Lasota, J. P. 2000, A\&A, 360, 575

Lasota, J. P. 2001, in Black Hole Binaries and Galactic Nuclei, ed. L. Kaper, E. P. J. van den Heuvel \& P. A. Woudt, Springer-Verlag, p149

Lasota, J. P. 2007, Comptes Rendus Physique, 8, 45

Lasota, J. P. 2008, New Astron. Rev., ed. M. A. Abramowicz (astro-ph/0801.0490)

Lasota, J. P., Abramowicz, M. A., Chen, X., Krolik, J., Narayan, R., \& Yi, I. 1996a, ApJ, 462, 142

Lasota, J. P., \& Hameury, J. P. 1998, AIP conf. Series, 431,351

Lasota, J. P., Narayan, R., \& Yi, I. 1996b, A\&A, 314,813

Li, L. X., Zimmerman, E. R., Narayan, R., \& McClintock, J. E. 2005, ApJS, 157, 335

Liu, B. F., Taam, R. F., Meyer-Hofmeister, E., \& Meyer, F. 2007, ApJ, 671, 695

Lobo, F. S. N. 2006, Class. Quantum Grav., 23, 1525

Loeb, A., Narayan, R., \& Raymond, J. C. 2001, ApJ, 547, L151

Loewenstein, M., Mushotzky, R. F., Angelini, L., Arnaud, K., \& Quataert, E. 2001, ApJ, 555, L21

Maccarone, T. C., \& Coppi, P. S. 2003, MNRAS, 338,189

Machida, M., Matsumoto, R., \& Mineshige, S. 2001, PASJ, 53, L1

Machida, M., Nakamura, K., \& Matsumoto, R. 2004, PASJ, 56, 671

Machida, M., Nakamura, K., \& Matsumoto, R. 2006, PASJ, 58, 193 
Mahadevan, R. 1997, ApJ, 477, 585

Mahadevan, R. 1998, Nature, 394, 651

Mahadevan, R., \& Quataert, E. 1997, ApJ, 490, 605

Malzac, J., Merloni, A., \& Fabian, A. C. 2004, MNRAS, 351, 253

Manmoto, T. 2000, ApJ, 534, 734

Manmoto, T., Mineshige, S., \& Kusunose, M. 1997, ApJ, 489, 791

Maraschi, L., \& Tavecchio, F. 2003, ApJ, 593, 667

Markoff, S., Falcke, H., \& Fender, R. P. 2001, A\&A, $372, \mathrm{~L} 25$

Markoff, S., Nowak, M. A., \& Wilms, J. 2005, ApJ, 635,1203

Mayer, M., \& Pringle, J. E. 2007, MNRAS, 376, 435

Mazur, P. O., \& Mottola, E. 2001, gr-qc/0109035

McClintock, J. E., Narayan, R., \& Rybicki, G. B. 2004, ApJ, 615, 402

McClintock, J. E., \& Remillard, R. A. 2006, in Black Hole Binaries, ed. W. Lewin \& M. van der Klis (Cambridge Univ. press) p157

McClintock, J. E., Shafee, R., Narayan, R., Remillard, R. A., Davis, S. W., \& Li, L. X. 2006, ApJ, 652,518

McKinney, J. C., \& Gammie, C. F. 2004, ApJ, 611, 977

McKinney, J. C. 2005, ApJ, 630, L5

McKinney, J. C. 2006, MNRAS, 368, 1561

Medvedev, M. V. 2000, ApJ, 541, 811

Meier, D. L. 2001, ApJ, 548, L9

Menou, K., Esin, A. A., Narayan, R., Garcia, M. R., Lasota, J. P., \& McClintock, J. E. 1999a, ApJ, 520, 276

Menou, K., Narayan, R., \& Lasota, J. P. 1999b, ApJ, 513,811

Menou, K., \& Quataert, E. 2001, ApJ, 552, 204

Merloni, A., Heinz, S., \& Di Matteo, T. 2003, MNRAS, 345, 1057

Meyer, F., Liu, B. F., \& Meyer-Hofmeister, E. 2000, A\&A, 361, 175

Meyer, F., \& Meyer-Hofmeister, E. 1994, A\&A, 361, 175

Meyer-Hofmeister, E., Liu, B. F., \& Meyer, F. 2005, A\&A, 432, 181

Miller, J. M., Homan, J., \& Miniutti, G. 2006a, ApJ, $652, \mathrm{~L} 113$

Miller, J. M., et al. 2006b, ApJ, 653, 525

Miller, J. M., et al. 2006c, Nature, 441, 953

Mitsuda, K., et al. 1984, PASJ, 36, 741

Miyamoto, S., et al. 1995, ApJ, 442, L13

Miyoshi, M., et al. 1995, Nature, 373, 127

Nagar, N. M., Falcke, H., Wilson, A. S., \& Ho, L. C. 2000, ApJ, 542, 186
Narayan, R. 1996, ApJ, 461, 136

Narayan, R. 2002, in Lighthouses of the Universe, ed. M. Gilfanov, R. Sunyaev (Springer) p405

Narayan, R. 2005, Astrophys. Sp. Sci., 300, 177

Narayan, R., Barret, D., \& McClintock, J. E. 1997a, ApJ, 482, 448

Narayan, R., Garcia, M. R., \& McClintock, J. E. 1997b, ApJ, 478, L79

Narayan, R., Garcia, M. R., \& McClintock, J. E. 2002a, in The Ninth Marcel Grossmann Meeting, ed. V. G. Gurzadyan, R. T. Jantzen \& R. Ruffini, World Scientific, p405

Narayan, R., \& Heyl, J. S. 2002, ApJ, 574, L139

Narayan, R., \& Heyl, J. S. 2003, ApJ, 599, 419

Narayan, R., \& Igumenshchev, I. V. 2000, ApJ, 539, 798

Narayan, R., Kato, S., \& Honma, F. 1997c, ApJ, 476,49

Narayan, R., Mahadevan, R., Grindlay, J. E., Popham, R. G., \& Gammie, C. 1998a, ApJ, 492, 554

Narayan, R., Mahadevan, R., \& Quataert, E. 1998b, in Theory of Black Hole Accretion Disks, ed. M. A. Abramowicz, G. Bjornsson, \& J. E. Pringle (Cambridge Univ. Press) p148

Narayan, R., \& McClintock, J. E. 2005, ApJ, 623, 1017

Narayan, R., McClintock, J. E., \& Yi, I. 1996, ApJ, 457,821

Narayan, R., Quataert, E., Igumenshchev, I. V., \& Abramowicz, M. A. 2002b, ApJ, 577, 295

Narayan, R., \& Yi, I. 1994, ApJ, 428, L13 (NY94)

Narayan, R., \& Yi, I. 1995a, ApJ, 444, 231 (NY95a)

Narayan, R., \& Yi, I. 1995b, ApJ, 452, 710 (NY95b)

Narayan, R., Yi, I., \& Mahadevan, R. 1995, Nature, 374,623

Nemmen, R. S., et al. 2006, ApJ, 643, 652

Noble, S. C., Leung, P. K., Gammie, C. F., \& Book, L. G. 2007, Class. Quant. Grav., 24, S259

Novikov, I. D., \& Thorne, K. S. 1973, in Blackholes, ed. C. DeWitt, \& B. DeWitt (Gordon \& Breach) p343

Orosz, J. A., Remillard, R. A., Bailyn, C. D., \& McClitnock, J. E. 1997, ApJ, 478, L83

Orosz, J. A., et al. 2007, Nature, 449, 872

Paczyński, B. 1998, Acta Astronomica, 48, 667

Pen, U. L., Matzner, C. D., \& Wong, S. 2003, ApJ, 596, L207

Perna, R., Narayan, R., Rybicki, G., Stella, L., \& Treves, A. 2003, ApJ, 594, 936

Piran, T. 1978, ApJ, 221, 652

Popham, R. G., \& Gammie, C. F. 1998, ApJ, 504, 
419

Popham, R. G., \& Narayan, R. 1991, ApJ, 370, 604 Quataert, E. 2001, in Probing the Physics of Active Galactic Nuclei by Multiwavelength Monitoring, ed. B. M. Peterson, R. S. Polidan \& R. W. Pogge (Astr. Soc. Pacific) p71

Quataert, E. 1998, ApJ, 500, 978

Quataert, E., Di Matteo, T., Narayan, R., \& Ho, L. C. 1999, ApJ, 525, L89

Quataert, E., \& Gruzinov, A. 1999, ApJ, 520, 248

Quataert, E., \& Gruzinov, A. 2000, ApJ, 539, 809

Quataert, E., \& Narayan, R. 1999, ApJ, 520, 298

Ramadevi, M. C., \& Seetha, S. 2007, MNRAS, 378, 182

Rees, M. J., Begelman, M. C., Blandford, R. D., \& Phinney, E. S. 1982, Nature, 295, 17

Remillard, R. A., Lin, D., Cooper, R. L., \& Narayan, R. 2006, ApJ, 646, 407

Reynolds, C. S., Di Matteo, T., Fabian, A. C., Hwang, U., \& Canizares, C. R. 1996, MNRAS, 283, L111

Rozanska, A., \& Czerny, B. 2000, A\&A, 360, 1170

Rykoff, E. S., Miller, J. M., Steeghs, D., \& Torres, M. A. P. 2007, ApJ, 666, 1129

Schödel, R., et al. 2002, Nature, 419, 694

Serabyn, E., Carlstrom, J., Lay, O., Lis, D. C., Hunter, T. R., \& Lacy, J. H. 1997, ApJ, 490, L77

Shahbaz, T., et al. 2005, MNRAS, 362, 975

Shakura, N. I., \& Sunyaev, R. A. 1973, A\&A, 24, 337

Shapiro, S. L., Lightman, A. P., \& Eardley, D. M. 1976, ApJ, 204, 187

Shapiro, S. L., \& Teukolsky, 1983, Black Holes, White Dwarfs, and Neutron Stars: The Physics of Compact Objects (Wiley-Interscience)

Sharma, P., Quataert, E., Hammett, G. W., \& Stone, J. M. 2007, ApJ, 667, 714

Sharma, P., Hammett, G. W., Quataert, E., \& Stone, J. M. 2006, ApJ, 637, 952

Shen, Z. Q., Lo, K. Y., Liang, M. C., Ho, P. T., \& Zhao, J. H. 2005, Nature, 438, 62

Sikora, M., Stawarz, L., \& Lasota, J. P. 2007, ApJ, 658,815

Smak, J. 1999, Acta Astronomica, 49, 391

Spruit, H. C., \& Deufel, B. 2002, A\&A, 387, 918

Spruit, H. C., Matsuda, T., Inoue, M., \& Sawada, K. 1987, MNRAS, 229, 517

Stolovy, S., Melia, F., McCarthy, D., \& Yusef-Zadeh, F. 2003, Astron. Nachr., 324, 419

Stone, J. M., \& Pringle, J. E. 2001, MNRAS, 322, 461

Stone, J. M., Pringle, J. E., \& Begelman, M. C. 1999, MNRAS, 310, 1002
Sunyaev, R. A., \& Titarchuk, L. G. 1980, A\&A, 86, 121

Tournear, D., et al. 2003, ApJ, 595, 1058

Ulvestad, J. S., \& Ho, L. C. 2001, ApJ, 562, L133

Visser, M., \& Wiltshire, D. L. 2004, Class. Quantum Grav., 21, 1135

Wu, X. 1997, MNRAS, 292, 113

Wu, X., \& Li, Q. 1996, ApJ, 469, 776

Wu, Q., Yuan, F., \& Cao, X. 2007, ApJ, 669, 96

Yuan, F. 2001, MNRAS, 324, 119

Yuan, F. 2003, ApJ, 594, L99

Yuan, F., \& Cui, W. 2005, ApJ, 629, 408

Yuan, F., Cui, W., \& Narayan, R. 2005, ApJ, 620, 905

Yuan, F., Ma, R., \& Narayan, R. 2008, ApJ, in press (astro-ph/08021679)

Yuan, F., \& Narayan, R. 2004, ApJ, 612, 724

Yuan, F., Quataert, E., \& Narayan, R. 2003, ApJ, 598,301

Yuan, F., \& Zdziarski, A. 2004, MNRAS, 354, 953

Yuan, F., Zdziarski, A., Xue, Y., \& Wu, X. B. 2007, ApJ, 659, 541

Yungelson, L. R., et al. 2006, A\&A, 454, 559

Zdziarski, A. A., \& Gierlinski, M. 2004, Prog. Theor. Phys. Suppl., 155, 99

Zdziarski, A. A., Lubinski, P., \& Smith, D. A. 1999, MNRAS, 303, L11

Zdziarski, A. A., Lubinski, P., Gilfanov, M., \& Revnivtsev, M. 2003, MNRAS, 342, 355

Zdziarski, A. A., et al. 2004, MNRAS, 351, 791

Zdziarski, A. A., Johnson, W. N., \& Magdiarz, P. 1996, MNRAS, 283, 193

Zdziarski, A. A., Poutanen, J., Mikolajewska, J., Gierlinski, M., Ebisawa, K., \& Johnson, W. L. 1998, MNRAS, 301, 435

Zhao, J. H., et al. 2003, ApJ, 586, L29

Zimmerman, E. R., Narayan, R., McClintock, J. E., \& Miller, J. M. 2005, ApJ, 618, 832 